\title{
The Towering Zeta Function
}

\author{
Michael M. Anthony
}

Enertron Corp., Hohenwald, TN, USA

Email: uinvent@aol.com

Received 18 November 2015; accepted 23 April 2016; published 26 April 2016

Copyright (C) 2016 by author and Scientific Research Publishing Inc.

This work is licensed under the Creative Commons Attribution International License (CC BY).

http://creativecommons.org/licenses/by/4.0/

(c) (i) Open Access

\section{Abstract}

Over a century and half has passed when Bernhard Riemann hypothesized that the non-trivial roots of the Riemann zeta function $\zeta(s)$ all lie on the half-line $s=\frac{1}{2}+i \sigma$. In this paper the Zeta function is iterated as a power tower and its properties are applied as an approach to an indication that the Riemann hypothesis might be true. It is known that complex valued Power towers converge under certain conditions to exponential power towers of entire functions. These properties can be used to resolve the Riemann Hypothesis.

\section{Keywords}

Riemann Hypothesis, Zeta, Power Towers, Convergence, Exponential Iterations

\section{Introduction}

The Zeta function seems to be the pyramid that holds the number systems together in a towering edifice of combinatorial relations.

Let $\mathbb{C}$ denote the complex numbers. They form a two-dimensional real vector space spanned by 1 and $i$ where $i$ is a fixed square root of -1 , and $x, y$ belong are real numbers, i.e. $\mathbb{C}=\{x+i y: x, y \in \mathbb{R}\}$. The Riemann Zeta function is a complex variable function defined as

$$
\zeta(s)=\frac{1}{1^{s}}+\frac{1}{2^{s}}+\frac{1}{3^{s}}+\cdots=\sum_{n=1}^{\infty} n^{-s}, s \in \mathbb{C}
$$

I will use the convention, $s=\sigma+i \tau$, where, $\tau \in \mathfrak{R}$ (reals).

Euler proved that the function $\zeta(s), s=\sigma+i t, \sigma>1$ can be represented in terms of primes, p. $\zeta(s)$ is analytic for $\sigma>1$ and satisfies in this half-plane the identity:

$$
\zeta(s)=\sum_{n=1}^{\infty} \frac{1}{n^{s}}=\prod_{p}^{\infty}\left(1-\frac{1}{p^{s}}\right)^{-1}
$$


Here, $p$ is a prime. Except for a pole at $s=1, \zeta(s)$ behaves properly and can be easily extended using the Gamma function. The extension of $\zeta(s)$ to the entire complex plane can be obtained by consideration the entirety and the general definition of the Gamma function:

$$
\Gamma(z)=\int_{0}^{\infty} \mathrm{e}^{-t} t^{z-1} \mathrm{~d} t
$$

Change variables by the substitution $t=n^{2} \pi x, \quad z=\frac{s}{2}$, in (3),

$$
\Gamma\left(\frac{s}{2}\right)=\left(n^{2} \pi\right)^{\frac{s}{2}} \int_{0}^{\infty} \mathrm{e}^{-n^{2} \pi x} x^{\frac{s}{2}-1} \mathrm{~d} x
$$

Extracting $\zeta(s)$ from(4),

$$
\pi^{-\frac{s}{2}} \zeta(s) \Gamma\left(\frac{s}{2}\right)=\sum_{n=1}^{\infty} \int_{0}^{\infty} \mathrm{e}^{-n^{2} \pi x} x^{\frac{s}{2}-1} \mathrm{~d} x
$$

The convergence of the series,

$$
S(x)=\sum_{n=1}^{\infty} \mathrm{e}^{-n^{2} \pi x}
$$

in the interval $[0, \infty]$ gives the relation:

$$
\pi^{-\frac{s}{2}} \zeta(s) \Gamma\left(\frac{s}{2}\right)=\int_{0}^{\infty} S(x) x^{\frac{s}{2}-1} \mathrm{~d} x
$$

This can be split into two separate integrals,

$$
\pi^{-\frac{s}{2}} \zeta(s) \Gamma\left(\frac{s}{2}\right)=\int_{0}^{1} S(x) x^{\frac{s}{2}-1} \mathrm{~d} x+\int_{1}^{\infty} S(x) x^{\frac{s}{2}-1} \mathrm{~d} x
$$

Note that the sum (6) is related to the Jacobi Theta function. See Ref. [1]

$$
\begin{gathered}
\theta(x)=\sum_{n=-\infty}^{\infty} \mathrm{e}^{-n^{2} \pi x} \\
2 S(x)=2 \sum_{n=1}^{\infty} \mathrm{e}^{-n^{2} \pi x}=\sum_{n=-\infty}^{\infty} \mathrm{e}^{-n^{2} \pi x}-1=\theta(x)-1
\end{gathered}
$$

The Jacobi theta function obeys the symmetry

$$
x^{\frac{1}{2}} \theta(x)=\theta\left(x^{-1}\right)
$$

Thus $x^{\frac{1}{2}}(2 S(x)+1)=2 S\left(x^{-1}\right)+1$,

$$
S\left(x^{-1}\right)=-\frac{1}{2}+\frac{1}{2} x^{\frac{1}{2}}+x^{\frac{1}{2}} S(x)
$$

The integral (8.0) now becomes

$$
\begin{gathered}
\pi^{-\frac{s}{2}} \zeta(s) \Gamma\left(\frac{s}{2}\right)=\int_{1}^{\infty} S\left(x^{-1}\right) x^{-\frac{s}{2}-1} \mathrm{~d} x+\int_{1}^{\infty} S(x) x^{\frac{s}{2}-1} \mathrm{~d} x \\
\pi^{-\frac{s}{2}} \zeta(s) \Gamma\left(\frac{s}{2}\right)=\int_{1}^{\infty} x^{-\frac{s}{2}-1}\left(-\frac{1}{2}+\frac{1}{2} x^{\frac{1}{2}}+x^{\frac{1}{2}} S(x)\right) \mathrm{d} x+\int_{1}^{\infty} S(x) x^{\frac{s}{2}-1} \mathrm{~d} x
\end{gathered}
$$




$$
\pi^{-\frac{s}{2}} \zeta(s) \Gamma\left(\frac{s}{2}\right)=\frac{1}{s(s-1)}+\int_{1}^{\infty} S(x)\left(x^{\frac{s}{2}-1}+x^{-\frac{s}{2}-\frac{1}{2}}\right) \mathrm{d} x
$$

The right side of the relation (15.0) is invariant to the substitution $s \rightarrow 1-s$. This gives the reflection formula for $\zeta(s)$ :

$$
\pi^{-\frac{s}{2}} \zeta(s) \Gamma\left(\frac{s}{2}\right)=\pi^{-\frac{1-s}{2}} \zeta(1-s) \Gamma\left(\frac{1-s}{2}\right)
$$

The reflection formula indicates that the roots should obey a reflection and conjugate symmetry if they lie on the $1 / 2$-line.

One can also study the maxima and minima of infinite products powers by looking at the functions that approximate the $\zeta$-function.

Proposition 2: The Zeta function is related to power towers.

Let $s=\sigma+i \tau$,

$$
\zeta(\sigma+i \tau)=\sum_{k=1}^{\infty} n^{-\sigma} \mathrm{e}^{-i \tau \ln (n)}=\sum_{k=1}^{\infty} n^{-\sigma}[\cos (\tau \ln (n))+i \sin (\tau \ln (n))]
$$

The $\zeta$ function can be written as a series in powers of $n$, where $n$ is an integer, in the form:

$$
\zeta(\sigma+i \tau)=\sum_{n=1}^{\infty}\left(r_{n}\right)^{n} \mathrm{e}^{i n \theta_{n}}
$$

where, $r_{n}=n^{-\frac{\sigma}{n}}, \theta_{n}=-\ln \left(n^{\frac{\tau}{n}}\right)$.

Note that in (21), as $\tau \rightarrow 0$ the minimum value of the terms for $\log \left(n^{-\frac{1}{n}}\right)$

$$
0, \log \left(\frac{1}{\sqrt{2}}\right), \log \left(\frac{1}{\sqrt[3]{3}}\right), \cdots, \log \left(\frac{1}{\sqrt[n]{n}}\right), \cdots
$$

is when $n=3$, but has repeated values for $n=2$, at $n=4$, since $2^{-\frac{1}{2}}=4^{-\frac{1}{4}}$. One can also write the Zeta function as follows:

$$
\zeta(\sigma+i \tau)=\sum_{n=1}^{\infty}\left(n^{-\frac{\sigma}{n}}\right)^{n}\left(n^{-\frac{i \tau}{n}}\right)^{n}
$$

From numerical calculations, the minima and maxima of the real and the complex parts of $\left(n^{-\frac{i \tau}{n}}\right)^{n}$ for $n>1$, occurs when $n=3$. This is due to the fact that $\frac{\ln 2}{2}=\frac{\ln 4}{4}$. One sees that something special is happening between these three points, $n=2, n=3$ and $n=4$ for the functions that define $\zeta(\sigma+i \tau)$. The graphs in Figure 1, Figure 2, and Figure 3 show the relationship of these points and they are tied together by the fact that $f(2)=f(4)$.

One can see that all three functions are somewhat related by the relation $s$ :

$$
f(x)\left\{\begin{array}{l}
=f(y) \quad x=2, y=2,4 \\
\neq f(y) \quad \text { otherwise }
\end{array}\right.
$$

This is true for the functions,

$$
f(n)=n^{-\frac{1}{n}}, g(x) \ln (x)=x \rightarrow
$$




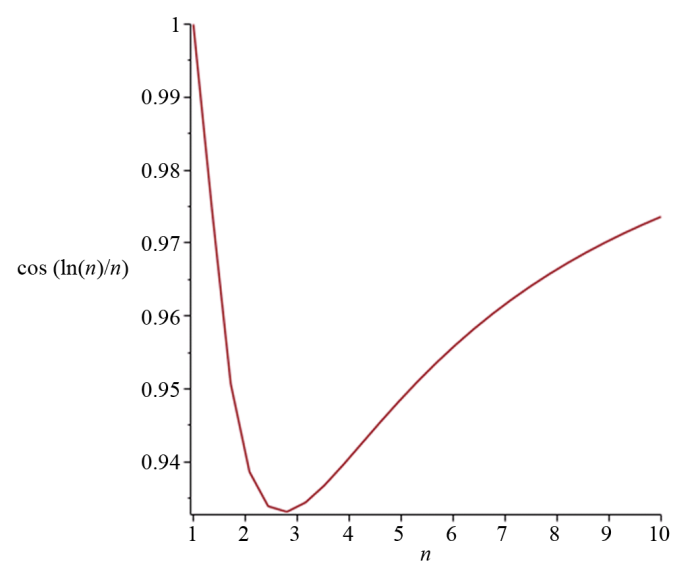

Figure 1. Shows that the minimum values of $\cos \left(\frac{\ln (n)}{n}\right)$ verus $n$ is close to $e$.

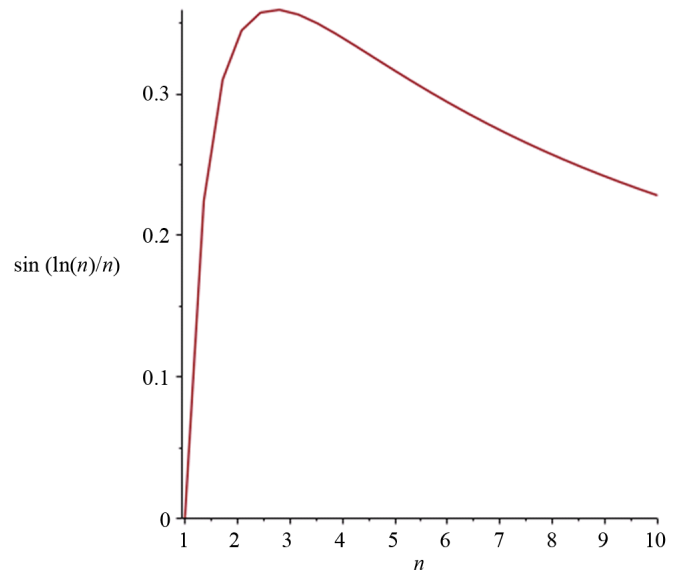

Figure 2. Shows the maximum value of $\sin (\ln (n) / n)$ is again exp (1).

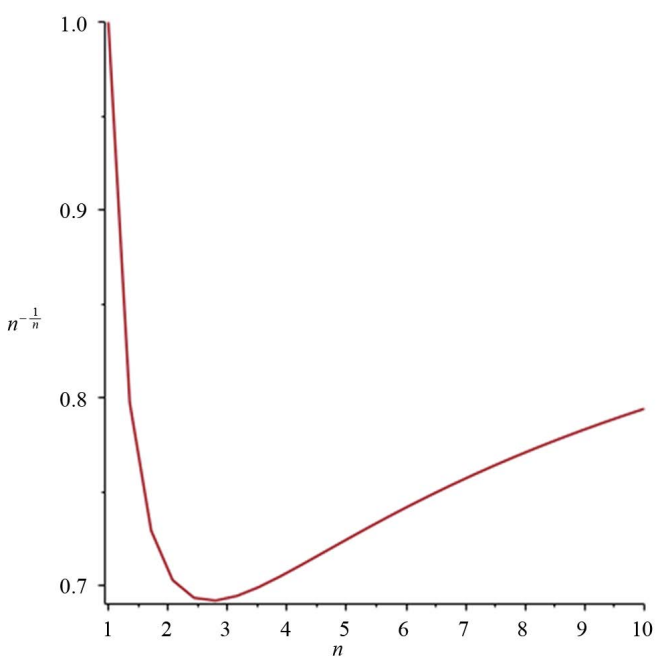

Figure 3. Shows the graphs of the real component $\left(n^{-\frac{1}{n}}\right)$ of the Zeta function. Again one sees that the minimum is at $\exp (1)$. 
One finds that these functions have their maxima and their minima in the range,

$$
\mathrm{e}^{-\frac{1}{\mathrm{e}}} \leq x \leq \mathrm{e}^{\mathrm{e}}
$$

This is exactly the range where real power towers converge. I will use this later when I discuss power towers.

\section{Relationship of the Riemann Zeta Function to Power Towers}

Power towers have been studied extensively. I start by describing power towers following some conventional methods that have been used by Knuth Ref. [2] and others.

\section{DEFINITION 1}

Let $n \in Z$. A power tower is defined as a follows:

$$
a \uparrow \uparrow n=a^{a^{a^{\uparrow}}}
$$

Here, I am using the Knuth notation for the tower of powers raised $n$ times. See Ref. [2].

\section{DEFINITION 2}

$$
g^{\bar{n}}(z)= \begin{cases}z & \text { if } n=0, \\ z^{z^{n-1}} & \text { if } n>1\end{cases}
$$

I have used the case $n=0$ as the argument itself, although in the literature most authors start with the definition $g^{\bar{n}}(z)=z, n=1$.

It is understood that such a power tower is iterated from some past argument to its present argument. By past I mean, the values that would have occurred in an iteration of the function that lead the iteration to its present value.

DEFINITION 3. If the limit of $z^{\bar{n}}$ exists then define it as follows:

$$
\lim _{n \rightarrow \infty} z^{\bar{n}}=z^{\bar{\infty}}
$$

DEFINITION 4. For $z \in \mathbb{C} \backslash\{x \in \mathbb{R}: x \leq 0\}$ and $n \in \mathbb{N}$, define the future power tower as the iterates starting from the present value $n=1$ as the first value that leads to a future $n^{\text {th }}$ value. Symbolically,

$$
g^{\vec{n}}(z)= \begin{cases}g(z) & \text { if } n=1 \\ g\left(g^{\overrightarrow{n-1}}(z)\right) & \text { if } n>1\end{cases}
$$

It is understood that such a power tower is iterated from its present state to some future value. By future I mean the argument values that will occur in an iteration of the function from its present value to some future value.

DEFINITION 5. The complex Lambert $W$ function $W(z)$ solves for $z$ the equation:

$$
W(z) \mathrm{e}^{W(z)}=z, \quad z \in \mathbb{C} .
$$

$W(z)$ is multi-valued and as has many branches with the usual notation for the $k^{\text {th }}$ branch as $W(k, z)$, with $k \in Z$ for the branch chosen. The principal branch of the function is $W(0, z)=W(z)$ and for real arguments, the function is denoted by $W(x)$. See Refs. [3]-[5]. The branch points of $W(k, z)$ take on real values only for $k \in Z=\{0,-1\}$. The function $W(k, z)$, satisfies, $W(0, z)>0$ for $x>0, W(0,0)=0$. The only other branch point that has real values is $W(-1, z)$ which lies in the range $(-\infty,-1), x \in\left[-\frac{1}{\mathrm{e}}, 0\right]$. Curves other than the semi-line, $(-\infty,-1)$, can be described by the parametric curves,

$$
C_{k}= \begin{cases}-\eta \cot \eta+i \eta, & \eta \in(2 \pi k,(2 k+1) \pi), k \geq 0 . \\ -\eta \cot \eta+i \eta, & \eta \in((2 k+1) \pi,(2 k+2) \pi), k<0\end{cases}
$$


These are subsets of the "Quadratrix of Hippias".

Since $\lim _{\eta \rightarrow 0}(\eta \cot \eta+i \eta)=-1$, the real values of the function occur at the branch points $(0,-1)$.

Consider the function,

$$
g(z)=z
$$

Let

$$
z=c^{z},
$$

LEMMA 1: The fixed points of $g(z)$ are $h(k, c)=\frac{W(k,-\log (c))}{-\log (c)}, k \in Z$.

Proof:

$$
\begin{gathered}
z \mathrm{e}^{-z \log c}=1, \\
-z \log y \mathrm{e}^{-z \log c}=-\log c .
\end{gathered}
$$

Using the Lambert $W$-function, $W(z) \mathrm{e}^{W(z)}=z$, for the $k^{\text {th }}$ branch point,

$$
Z=\frac{W(k,-\log (c))}{-\log (c)}, k \in Z
$$

Obviously, $z$ is constant over the range of values of $c$ that satisfy the relation (31), thus the fixed points of the function $g(z)$ are,

$$
h(k, y)=\frac{W(k,-\log (c))}{-\log (c)}, k \in Z .
$$

If $c \in \mathbb{C}, c \notin\{0,1\}$, the sequence $\left\{g^{\vec{k}}(z)\right\}_{k \in \mathbb{N}}$ converge to fixed points that satisfy (32) and exponential function of $z$, as will be seen later. For real values of $x$, the function $g(x)$ only converges within the range of validity

$$
\mathrm{e}^{-\frac{1}{\mathrm{e}}} \leq x \leq \mathrm{e}^{\mathrm{e}} \text {, i.e. } 0.6922006275 \leq x \leq 15.154 \cdots
$$

How does this relate to the iterations of the Zeta function?

DEFINITION 6: Define an iterated exponential as the Towering Zeta function:

$$
\zeta^{\vec{n}}(z)=\zeta(\zeta(\zeta \cdots(\zeta(z))))
$$

Here, $\zeta^{\vec{n}}$ is defined as a future iterated exponential by taking the $\zeta$-value of (z) and then taking the $\zeta$-value of $\zeta(\zeta(z))$ and repeating this process $n$-times. The arrow shows the direction of iteration of Towering Zeta functions and the arrow $\rightarrow$ means take increasing nests of $\zeta$ values of prior Zeta values to obtain a new future value.

DEFINITION 7: Define the inverse past iterated exponential of the Towering Zeta function $\zeta(z), \operatorname{Re}(z)>1$, as follows:

$$
\zeta^{\overline{\bar{n}}}(z)=\zeta^{-1}\left(\zeta^{-1}\left(\zeta^{-1} \cdots\left(\zeta^{-1}(z)\right)\right)\right)
$$

Here the arrow $\leftarrow$ means take decreasing past nests of $\zeta^{-1}$ where the inverse-zeta function is one of the set of infinite solutions to the equation, $\zeta(x)=z$ and so

$$
\zeta^{-1}(z)=x \in C .
$$


Since the solutions to (36) are multivalued, we wish to fix particular solutions that will revert the function values back to original values from its inverse values.

Start with the reflection formula:

$$
\begin{gathered}
\pi^{-\frac{s}{2}} \zeta(s) \Gamma\left(\frac{s}{2}\right)=\pi^{-\frac{1-s}{2}} \zeta(1-s) \Gamma\left(\frac{1-s}{2}\right) \\
\pi^{-\frac{s}{2}} \zeta(s) \Gamma\left(\frac{s}{2}\right)=\pi^{-\frac{1-s}{2}} \zeta(1-s) \Gamma\left(\frac{1-s}{2}\right)=K
\end{gathered}
$$

where,

$$
K=\sum_{n=1}^{\infty} \int_{0}^{\infty} \mathrm{e}^{-n^{2} \pi x} x^{\frac{s}{2}-1} \mathrm{~d} x
$$

Let there be solutions $\left\{z_{s}, z_{1-s}\right\} \in \mathbb{C}$, such that

$$
\begin{aligned}
& \zeta(s)=\frac{K \pi^{\frac{s}{2}}}{\Gamma\left(\frac{s}{2}\right)}, \rightarrow \zeta^{-1}\left(\frac{K \pi^{\frac{s}{2}}}{\Gamma\left(\frac{s}{2}\right)}\right)=z_{s}, s \in z_{s} \\
& \zeta(1-s)=\frac{K \pi^{\frac{1-s}{2}}}{\Gamma\left(\frac{1-s}{2}\right)}, \zeta^{-1}\left(\frac{K \pi^{\frac{1-s}{2}}}{\Gamma\left(\frac{1-s}{2}\right)}\right)=z_{1-s}, 1-s \in z_{1-s}
\end{aligned}
$$

Then the infinite number of possible inverse-solutions are paired as follows:

$$
z_{s}+z_{1-s}=1
$$

Then these particular solutions of the function obeys the rules:

$$
\begin{gathered}
\zeta^{\bar{n}}\left(\zeta^{\overline{\bar{n}}}\left(z_{s}\right)\right)=z_{s}, \\
\zeta^{\overline{m-n}}\left(z_{s}\right), m>n \\
\zeta^{\vec{m}}\left(\zeta^{\bar{n}}\left(z_{s}\right)\right)=\zeta^{\overline{\bar{n}}}\left(\zeta^{\vec{m}}\left(z_{s}\right)\right)=z_{s}, m=n \\
\zeta^{\overline{n-m}}\left(z_{s}\right), n>m \\
\zeta^{\vec{n}}\left(z_{s}\right)=\zeta^{\overline{n-k}}\left(\zeta^{\vec{k}}\left(z_{s}\right)\right)
\end{gathered}
$$

For example, if only consider $z \in\left\{z_{s}=\sigma_{0}+i \tau_{0}\right\}$, then there exists a sequence of constants $\sigma_{n}+i \tau_{n}$ such that

$$
\begin{aligned}
\zeta^{\overrightarrow{1}}\left(\sigma_{0}+i \tau_{0}\right) & =\zeta\left(\sigma_{0}+i \tau_{0}\right)=\sigma_{1}+i \tau_{1} \\
\zeta^{\overrightarrow{2}}\left(\sigma_{0}+i \tau_{0}\right) & =\zeta\left(\zeta\left(\sigma_{0}+i \tau_{0}\right)\right)=\zeta\left(\sigma_{1}+i \tau_{1}\right)=\sigma_{2}+i \tau_{2} \\
\zeta^{\overrightarrow{3}}\left(\sigma_{0}+i \tau_{0}\right) & =\zeta\left(\zeta^{\overrightarrow{2}}\left(\sigma_{0}+i \tau_{0}\right)\right)=\zeta \zeta\left(\sigma_{1}+i \tau_{1}\right)=\zeta\left(\zeta\left(\zeta\left(\sigma_{0}+i \tau_{0}\right)\right)\right)=\zeta\left(\sigma_{2}+i \tau_{2}\right)=\sigma_{3}+i \tau_{3} \\
& \vdots \\
\zeta^{\vec{n}}\left(\sigma_{0}+i \tau_{0}\right) & =\zeta\left(\zeta^{\overline{n-1}}\left(\zeta^{\overline{n-2}}\left(\cdots\left(\zeta\left(\sigma_{0}+i \tau_{0}\right)\right)\right)\right)\right)
\end{aligned}
$$

One can expand these functions as follows: 


$$
\begin{aligned}
\zeta^{\overrightarrow{2}}(z) & =1^{-\zeta^{\overline{1}}(z)}+2^{-\zeta^{\underline{1}}(z)}+3^{-\zeta^{\overline{1}}(z)}+\cdots \\
& =1^{1^{-(z)}-2^{-(z)}-3^{-(z)}+\cdots}+2^{-1^{-(z)}-2^{-(z)}-3^{-(z)}+\cdots}+3^{1^{-(z)}-2^{-(z)}-3^{-(z)}+\cdots}+\cdots \\
& =1+2^{-1^{-(z)}} 2^{-2^{-(z)}} 2^{-3^{-(z)}}+\cdots+3^{-1^{-z}} 3^{-2^{-(z)}} 3^{-3^{-(z)}}+\cdots \\
& \zeta^{\overrightarrow{2}}(z)=\sum_{a_{2}=2 a_{1}=1}^{\infty} \prod^{\infty}\left(a_{2}^{-a_{1}^{-z}}\right)
\end{aligned}
$$

As another general example,

$$
\begin{gathered}
n=5, \quad \sum \text { form } \\
\zeta^{\overrightarrow{5}}(z)=\sum_{a_{5}=1}^{\infty} a_{5}\left(\sum_{a_{4}=1 a_{4}}^{-\sum_{a_{3}=1}^{\infty} a_{3}-\sum_{a_{2}=1}^{\infty} a_{2}-\sum_{a_{1}=1}^{\infty} a^{a_{1}^{-z}}}\right)
\end{gathered}
$$

This can be converted to a product form:

$$
\begin{gathered}
n=5, \quad \prod \text { form } \\
\zeta^{\overrightarrow{5}}(z)=\sum_{a_{5}=1 a_{4}=1}^{\infty} a_{5}^{\infty} \prod_{a_{3}=1}^{\infty a_{4}}{ }^{-\prod_{a_{2}=1}^{\infty} a_{3}}-\prod_{a_{1}=1}^{\infty} a^{a_{2}-a_{1}^{-z}}
\end{gathered}
$$

Here, $a_{0}, a_{1}, \cdots, a_{n}=1,2,3, \cdots, \infty$ are independent integers. In general,

$$
\zeta^{\vec{n}}(z)=\sum_{a_{n}=1 a_{n-1}=1}^{\infty} \prod_{n}^{-\prod_{a_{n-2}=1}^{\infty} a_{n-1}-\prod_{a_{n-3}=1}^{\infty} a_{n-2}-\cdots-\Pi_{a_{1}=1}^{\infty} a_{2}^{-}-\prod_{a_{0}=1}^{\infty} a_{1}^{a_{0}^{-}} a^{-z}}
$$

Thus, taking

$$
Z_{n}=\prod_{a_{n-1}=1}^{\infty} a_{n}^{-z_{n-1}}=a_{n}^{-\sum_{a_{n-1}=1}^{\infty} z_{n-1}}
$$

Then,

$$
\zeta^{\vec{n}}(z)=\sum_{a_{n}=1}^{\infty} z_{n}
$$

Thus, $\zeta^{\vec{n}}(z)$ is a power tower that can be put in the general form:

$$
\zeta^{\vec{n}}(z)=\sum_{a_{n}=1}^{\infty} g^{\vec{n}}(z)
$$

This however is only valid for $\mathfrak{R}(z)>1$.

Now consider the same power towers in terms of primes.

In terms of primes, let $p_{b_{0}}$ be the $b_{1}^{\text {th }}=1$, prime with $p_{1}=2$. Then,

$$
\begin{gathered}
\zeta^{\overrightarrow{1}}(z)=\prod_{b_{1}=1}^{\infty}\left(1-\frac{1}{p_{b_{1}}{ }^{2}}\right)^{-1}=\prod_{b_{1}=1}^{\infty}\left(\frac{p_{b_{1}}{ }^{z}}{p_{b_{1}}{ }^{2}-1}\right)=\prod_{b_{1}=1 a_{1}=0}^{\infty} \sum_{b_{1}}^{\infty} p^{-a_{1} z} \\
\zeta^{\overrightarrow{2}}(z)=\prod_{b_{2}=1 a_{2}=0}^{\infty} \sum^{\infty}\left(p_{b_{2}}{ }^{-a_{2} \prod_{b_{1}=0}^{\infty} \Sigma_{a_{1}=0}^{\infty}\left(p_{b_{1}}{ }^{-a_{1} z}\right)}\right)
\end{gathered}
$$




$$
\left.\zeta^{\vec{n}}(z)=\prod_{b_{n}=1 a_{n}=0}^{\infty} \sum^{\infty}\left(p_{b_{n}}{ }^{-a_{n} \prod_{b_{n-1}=1}^{\infty} \sum_{a_{n-1}=0}^{\infty}\left(p_{b_{n-1}}-a_{n-1} \Pi_{b_{n-2}=1}^{\infty} \sum_{a_{n-2}=0}^{\infty}\left(p_{b_{n-2}}-a_{n-2} \cdots\right)\right.}\right)\right)
$$

Thus, taking

$$
P_{n}(z)=\prod_{b_{n}=1 a_{n}=0}^{\infty} \sum_{b_{n}}{ }^{-a_{n} P_{n-1}(z)}=\zeta\left(P_{n-1}(z)\right)
$$

The Zeta power tower in terms of primes becomes the simple form:

$$
\zeta^{\vec{n}}(z)=P^{\vec{n}}(z)=\prod_{b_{n}=1 a_{n}=0}^{\infty} p_{b_{n}}^{-a_{n} p^{n-1}(z)}
$$

3. Convergence of $\zeta^{\vec{n}}(z)$ for $1>\Re(z)>0$

Consider the extended Zeta function, $\mathfrak{R}(z)>0$.

$$
\zeta(z)=\frac{1}{1-2^{1-z}} \sum_{a_{0}=1}^{\infty} a_{0}^{-z}(-1)^{a_{0}}
$$

Then,

$$
\zeta(z)=\sum_{b_{0}=1 a_{0}=1}^{\infty}(-1)^{a_{0}} a_{0}^{-z} 2^{b_{0}(1-z)}
$$

Using this,

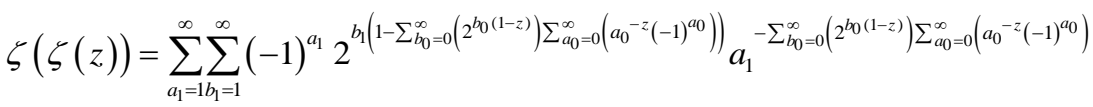

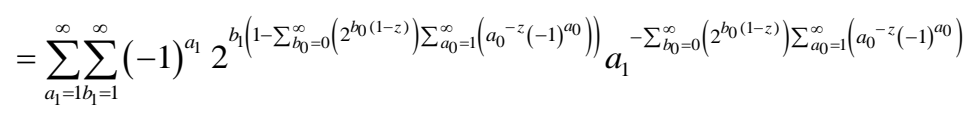

$$
\begin{aligned}
& =\sum_{a_{1}=1 b_{1}=1}^{\infty}(-1)^{a_{1}} 2^{b_{1}} \prod_{b_{0}=1 a_{0}=1}^{\infty}\left(2^{b_{1}} a_{1}\right)^{\left(-(-1)^{a_{0} 2_{0}(1-z)} a_{0}-z\right)}
\end{aligned}
$$

Further,

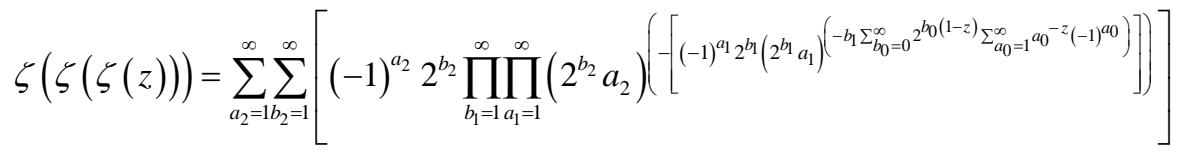

$$
\begin{aligned}
& =\sum_{a_{2}=1 b_{2}=1}^{\infty} \sum^{\infty}\left[(-1)^{a_{2}} 2^{b_{2}} \prod_{b_{1}=1 a_{1}=1}^{\infty} \prod^{\infty}\left(2^{b_{2}} a_{2}\right)\left(\left[(-1)^{a_{1} 2^{b_{1}}} \prod_{b_{0}=1}^{\infty} \prod_{a_{0}=1}^{\infty}\left(2^{b_{1}} a_{1}\right)\left(\left[(-1)^{\left.a_{0} b_{1} 2^{b_{0}}\left(2^{b_{0}} a_{0}\right)^{\left(-b_{0_{2}}\right)}\right]}\right]\right)\right]\right)\right]
\end{aligned}
$$

Thus, taking,

$$
Z_{n}=(-1)^{a_{n}} 2^{b_{n}} \prod_{b_{n-1}=1 a_{n-1}=1}^{\infty} \prod^{\infty}\left(2^{b_{n}} a_{n}\right)^{\left(-Z_{n-1}\right)}
$$

The iterated zeta function becomes:

$$
\zeta^{\vec{n}}(z)=\sum_{a_{n}=1 b_{n}=1}^{\infty} \sum_{n}
$$


Note that

$$
\begin{aligned}
& \zeta^{\vec{n}}(z)=\sum_{a_{n}=1 b_{n}=1}^{\infty} Z_{n}=\sum_{a_{n}=1 b_{n}=1}^{\infty} \sum^{\infty}(-1)^{a_{n}} 2^{b_{n}} \prod_{b_{n-1}=1 a_{n-1}=1}^{\infty} \prod^{\infty}\left(2^{b_{n}} a_{n}\right)^{\left(-Z_{n-1}\right)}
\end{aligned}
$$

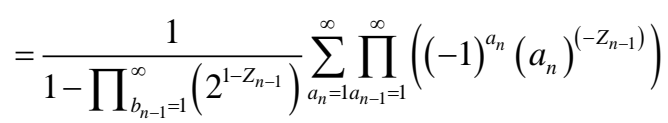

$$
\begin{aligned}
& =\frac{1}{1-2^{1-\sum_{a_{n-1}=1}^{\infty} \sum_{b_{n-1}=1}^{\infty} Z_{n-1}}} \sum_{a_{n}=1}^{\infty}(-1)^{a_{n}}\left(a_{n}\right)^{\left(-\sum_{a_{n-1}=1}^{\infty} \sum_{b_{n-1}=1}^{\infty} Z_{n-1}\right)} \\
& \zeta^{\vec{n}}(z)=\zeta\left(\zeta^{\overline{n-1}}(z)\right)=\frac{1}{1-2^{1-\zeta^{n-1}}(z)} \sum_{a_{n}=1}^{\infty}(-1)^{a_{n}}\left(a_{n}\right)^{\left(-\zeta^{\overline{n-1}}(z)\right)}
\end{aligned}
$$

This is just the relation for the general zeta function:

$$
\zeta(z)=\frac{1}{1-2^{1-z}} \sum_{a_{n}=1}^{\infty}(-1)^{a_{n}}\left(a_{n}\right)^{(-z)}
$$

One sees that this is also a power tower product but this time the sum and the product operators are doubled. Thus the iterated exponential power tower of the zeta function for $\mathfrak{R}(z)>0$ can be separated into the product of two power towers:

$$
\begin{aligned}
Z_{n} & =(-1)^{a_{n}} 2^{b_{n}} \prod_{b_{n-1}=1 a_{n-1}=1}^{\infty} \prod^{\infty}\left(2^{b_{n}} a_{n}\right)^{\left(-Z_{n-1}\right)} \\
& =\mathrm{e}^{\pi i a_{n}} 2^{b_{n}} \prod_{b_{n-1}=1 a_{n-1}=1}^{\infty} \prod^{\infty}\left(2^{b_{n}} a_{n}\right)^{\left(-Z_{n-1}\right)}
\end{aligned}
$$

Since the raising power is not over the entire product, this becomes difficult to write in the general Knuth form. The future iterates of the function $\zeta^{\vec{\infty}}(z)$ converges to a constant whenever there exists some root of the Towering Zeta function, $s_{r}$ that satisfies $\zeta^{\vec{r}}(z)=s_{r}, m<\infty$. Thus, all the arguments that lead to a root are unique and no periods of the function can exist in the past iterations. This does not exclude close to periodic regions. This subject has been extensively studied in Julia Sets theory.

$$
\zeta^{\overline{r+1}}(z)=\sum_{n=1}^{\infty} n^{-\zeta^{\vec{r}}(z)=s_{r}}=0
$$

Let $\zeta^{\vec{r}}\left(z \in Z_{s}\right)=s_{r}$ be a root of the Zeta function. Then, the future iterates $\zeta^{\overline{r+k}}(z), k=1, \cdots, \infty$, converge toconstants over all arguments.

$$
\zeta^{\overrightarrow{r+1}}(z)=\zeta\left(\zeta^{\vec{r}}(z)\right)=\zeta\left(s_{r}\right)=0
$$

Obviously, the function vanishes for $1-s_{r}$ also.

When the function is continued over values of $k$ for a given root, $s_{r}$,

$$
\begin{aligned}
& \zeta^{\vec{r}}(z)=s_{r} \\
& \zeta^{\overline{r+1}}(z)=\zeta\left(s_{r}\right)=0 \\
& \zeta^{\overline{r+2}}(z)=\zeta(0)=-\frac{1}{2}, \\
& \zeta^{\overline{r+13}}(z)=\zeta\left(-\frac{1}{2}\right)=-0.2078862250 \cdots, \\
& \zeta^{\overline{r+4}}(z)=\zeta(-0.2078862250 \cdots)=-0.3448741787 \cdots, \\
& \quad \vdots \\
& \zeta^{\vec{\infty}}(z)=\zeta(-0.2959050806 \cdots)=-0.2959050806 \cdots .
\end{aligned}
$$


This can be seen when the function is taken to the limit, and becomes an image of itself.

$$
\lim _{n \rightarrow \infty} \zeta^{\vec{k}}(z)=\zeta\left(c_{\infty}\right)=\zeta\left(c_{\infty-1}\right)=c_{\infty}=\text { Constant. }
$$

The solutions to the relation $\zeta(x)=x$, for can be obtained if one takes

$$
\sum_{a_{\infty}=1}^{\infty} a_{\infty}{ }^{-c_{\infty}}=c_{\infty}
$$

Thus $\zeta^{\vec{\infty}}(x)$ converges for real values when $n$ is large and obviously the power tower product converges to the constant function,

$$
\zeta^{\vec{\infty}}(x)=\zeta\left(\zeta^{\overline{\infty-1}}(x)\right) \rightarrow \zeta\left(c_{\infty}\right)=c_{\infty} \approx-0.295905005575214 \cdots
$$

If there appears an argument $\zeta^{\vec{r}}(z)=s_{r}, r<\infty$, then $\zeta\left(c_{\infty}\right)=c_{\infty}$ is the fixed point and the real limit of the function $\zeta^{\infty}(z)$, when $s_{r}$ is a root of the Zeta function. The convergence becomes real and the complex part of the arguments vanish. A plot of these values for $\zeta^{\overline{k+1}}\left(s_{r}\right)=0$ is shown in Figure 4 for the converging values. Thus $C_{\infty}$ is a super attractor for all roots of the Zeta function.

Any roots $s_{r}$ of the Zeta function that appears in any iteration of the function at the $r^{\text {th }}$ term, $r<n$, will converge the function, $\zeta^{\vec{n}}(z)$. The functions of the infinite iterative form:

$$
\zeta^{\vec{\infty}}(\sigma+i \tau)=\zeta(\zeta(\zeta \cdots(\zeta(\sigma+i \tau))))
$$

have certain attracting and repelling values, and are sometimes periodic with respect to $n$. If one takes roots $s_{r}$ of the function as a starting point one finds that the function generates constants for each value of $n$, such that:

$$
\begin{aligned}
\zeta^{\overrightarrow{0}}\left(s_{r}\right) & =c_{0}=s_{r} \\
\zeta^{1} & \left(s_{r}\right)=\zeta\left(s_{r}\right)=c_{1}=0 \\
\zeta^{\overrightarrow{2}}\left(s_{r}\right) & =\zeta\left(\zeta\left(s_{r}\right)\right)=c_{2}=-\frac{1}{2}, \\
\zeta^{\overrightarrow{3}}\left(s_{r}\right) & =\zeta\left(-\frac{1}{2}\right)=c_{3}=-0.2078862250 \cdots, \\
\zeta^{\overrightarrow{4}}\left(s_{r}\right) & =\zeta(-0.2078862250 \cdots)=c_{4}=-0.3448741787 \cdots, \\
& \vdots \\
\zeta^{\vec{\infty}}\left(s_{r}\right) & =c_{\infty}=-0.2959050806 \cdots .
\end{aligned}
$$

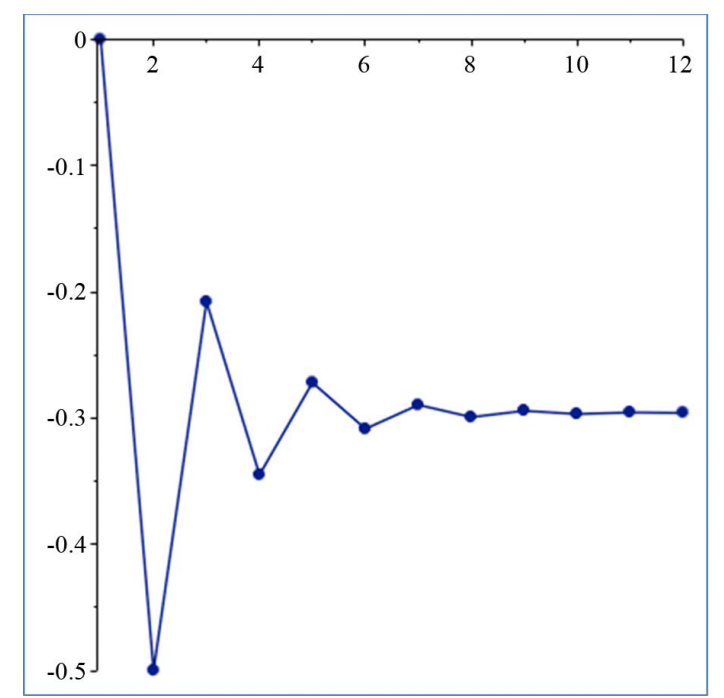

Figure 4. Shows the oscillations for convergence of iterates from a root. 
Then, for all roots $s_{r}$ of the zeta function, there exists a unique sequence of real constants that are invariant with respect to all the roots and that converge to the same value, $c_{\infty}$. The plots below show examples of how the function's values change for the real arguments generated by some complex roots. $\Re\left(\zeta^{\vec{n}}\left(s_{r}\right)\right)$, is the vertical axis as $n$ changes (horizontal axis) for various indicated values of $\tau=2,3,4,14.1347251$ and 21.0220396. One can see that the function has alternating maxima and minima at integer values of $n$ with the lowest value occurring again at $n=3$. This is again due to that fact that the function converges to a constant for all roots as $n \rightarrow \infty$.

One can surmise that any root $s_{r}$ such that $\zeta\left(s_{r}\right)$ vanishes will generate a convergent sequence of real arguments $s_{k}$, for the functions $\zeta^{\vec{k}}\left(s_{k}\right), k>0$, such that

$$
\Re\left(s_{r}\right)>c_{\infty}>\zeta(0)=-\frac{1}{2} .
$$

Assuming the Riemann Hypothesis, there is a symmetry between the real parts of the known complex roots $s$, of the function about zero:

$$
\mathfrak{R}\left(+\frac{1}{2} \pm i t\right)=\frac{1}{2} \rightarrow \zeta\left(\frac{1}{2} \pm i t\right)=0 \rightarrow \zeta(0)=-\frac{1}{2}
$$

This symmetry is due to the fact that at any point in a large number of iterations where a root $s_{r}$, one could replace zero of $\zeta\left(s_{r}\right)$ with the equivalence, $0 \equiv \zeta\left(s_{r}\right)$;

$$
\begin{gathered}
\zeta(\zeta(\zeta \cdots(\zeta(0)))) \rightarrow \zeta(\zeta(\zeta \cdots(0))), n \rightarrow \infty \\
\left.\zeta\left(\zeta\left(\zeta \cdots \zeta\left(\zeta\left(\frac{1}{2} \pm \mathrm{it}\right)\right)\right)\right)\right) \rightarrow \zeta(\zeta(\zeta \cdots \zeta(0))) \rightarrow \zeta\left(\zeta\left(\zeta \cdots\left(-\frac{1}{2}\right)\right)\right), n \rightarrow \infty
\end{gathered}
$$

This implies that the convergence of the iterations of functions must be symmetric about a zero of the function as implied by the mean value theorem mention earlier.

It is worth noting that the complex parts of iterates of the complex arguments result in real arguments if the products of the components of the iterates is real. Thus the roots are expected to be composed of a spectrum of complex factors whose iterates are real since they result in a quadratic convergence to the real values due to the symmetry of the reflection formula.

\section{Convergence of the Riemann-Zeta Function for Complex Values}

Let $\mathrm{Z}$ belong to integers and $\mathrm{Z}^{+}$to the subset of positive integers. Any sequence of arguments can be created by functional iteration. Let function $g(z): \mathbb{C} \rightarrow \mathbb{C}$, with an initial value $z_{0}$. The sequence obtained $\left\{z_{0}, z_{1}=g\left(z_{0}\right), \cdots, z_{m-1}=g\left(z_{m}\right), \cdots\right\}$ can yield periodic $m$-cycles $g^{\vec{m}}\left(z_{c}\right)=z_{c}$ for some $z_{c} \in \mathbb{C}$. A critical periodic point $z_{c}$ can be classified depending on the value of the derivatives, $\chi_{c}=\left(g^{\vec{m}}\left(z_{c}\right)\right)$. Such points are studied in Fractals, Chaos theory, Attracting Periodic Cycles, and in Mandelbrot Sets, using Newton approximations and this critical point is classified as

$$
\begin{aligned}
& \text { Superattracting if }\left|\chi_{c}\right|=0 \\
& \text { Attracting if }\left|\chi_{c}\right|<1 \\
& \text { Neutral if }\left|\chi_{c}\right|=1 \\
& \text { Repelling if }\left|\chi_{c}\right|>1
\end{aligned}
$$

Let $\left\{z_{0}, z_{1}, \cdots, z_{k}-1\right\}$ be an $k$-cycle of the differentiable function $g^{\vec{n}}\left(z_{0}\right)$, with $z_{k}=g^{\vec{k}}\left(z_{0}\right)=g\left(z_{k-1}\right)$. Then, from definition (7),

$$
\left(g^{\bar{n}}\right)^{\prime}(z)=\left([\log c]^{n}\right) \prod_{k=1}^{n} g^{\vec{k}}(z)
$$

Then, the function $g^{\vec{n}}(z)$ has the following properties. 
- If $z=g^{\vec{p}}(z)$ is a periodic point of period $m$, then $g^{\vec{m}}(z)=z$, if and only if $m$ divides $p$. This product is referred to as the multiplier of a periodic point $z$ of period $p$. Since the $z_{i}$ lie on a cycle,

$$
\left(g^{k}\right)^{\prime}\left(z_{i}\right)=\left(g^{k}\right)^{\prime}\left(z_{0}\right) \text { for all } i \text {. }
$$

- If $z=g^{\vec{p}}(z)$ is a periodic point of period $m$, then, $\zeta^{\vec{p}}(z)=\zeta^{\vec{q}}(z)$ if and only if $m$ divides $p-q$.

LEMMA 2: If $s$ is a root of the Zeta function, then the Towering Zeta function, $\zeta^{\bar{m}}(z) \neq \zeta(s)$ for finite values of $m>1$, unless either $z \in\left[c_{m}\right]$, or $z=s$ when $m=1$.

Proof: The case $m=1$ is trivial for $\zeta^{1}(z)=\zeta(s)=0$. Suppose there exists some $\zeta^{\bar{r}}(z)=c_{r}$, where $r$ is a positive integer. Then there exists some

$$
\zeta\left(\zeta^{\overline{r-1}}(z)\right)=c_{r}
$$

i.e. there exists a sequence of arguments,

$$
\begin{aligned}
\zeta^{\overrightarrow{0}}\left(s_{r}\right) & =c_{0}=s_{r} \\
\zeta^{\overrightarrow{1}}\left(s_{r}\right) & =\zeta\left(s_{r}\right)=c_{1}=0 \\
\zeta^{\overrightarrow{2}}\left(s_{r}\right) & =\zeta\left(\zeta\left(s_{r}\right)\right)=c_{2}=-\frac{1}{2}, \\
\zeta^{\overrightarrow{3}}\left(s_{r}\right) & =\zeta\left(-\frac{1}{2}\right)=c_{3}=-0.2078862250 \cdots \\
\zeta^{\overrightarrow{4}}\left(s_{r}\right) & =\zeta(-0.2078862250 \cdots)=c_{4}=-0.3448741787 \cdots, \\
\vdots & \\
\zeta^{\overrightarrow{0}}\left(s_{r}\right) & =c_{\infty}=-0.2959050806 \cdots \\
\zeta\left(\zeta\left(\zeta^{\overline{r-2}}\left(c_{r-2}\right)\right)\right) & =\zeta\left(\zeta\left(\zeta\left(\zeta^{\overline{r-3}}\left(c_{r-3}\right)\right)\right)\right)=\zeta\left(\zeta\left(\zeta\left(\zeta \cdots \zeta\left(\zeta^{\overrightarrow{0}}\left(c_{0}\right)\right)\right)\right)\right)=s,
\end{aligned}
$$

Then,

$$
z=\zeta\left(\zeta\left(\zeta \cdots \zeta\left(\zeta^{\overrightarrow{0}}(z)\right)\right)\right) \in\left[c_{m}\right]
$$

The iterated exponential that generates a root $\zeta^{\vec{r}}\left(c_{0}\right)=0$, must satisfy all roots independently, since if for some $r, z_{r}$ is a root of $\zeta^{\bar{m}}(z)$, then $z_{r}$ must generate a particular sequence of real arguments in a future sequence of arguments that must converge to $c_{\infty}$, as $k \rightarrow \infty$, i.e., $\zeta^{\overrightarrow{0}}\left(z_{0}\right)=z_{0}, \zeta^{\overrightarrow{1}}\left(z_{0}\right)=z_{1}$,

$\zeta^{\overrightarrow{1}}\left(z_{0}\right)=z_{2}, \cdots, \zeta^{\overline{r-2}}\left(z_{0}\right)=z_{r-1}, \zeta^{\overline{r-1}}\left(z_{0}\right)=z_{r}, \quad \zeta^{\vec{r}}\left(z_{0}\right)=0, \zeta^{\overline{r+1}}\left(z_{0}\right)=-\frac{1}{2}, \cdots \rightarrow c_{\infty}$. Such a sequence of real arguments cannot generate another complex root in the future direction. As an aside, Little wood showed that if the sequence $z_{k}, k=1, \cdots, r$, contains all the imaginary parts of all zeros in the upper half-plane in ascending order, then,

$$
\lim _{k \rightarrow \infty} z_{k+1}-z_{k}=0
$$

There cannot exist such a sequence of roots in the past or future arguments of $\zeta^{\vec{n}}\left(z_{0}\right)$ since when a root is encountered the sequence of arguments converge and never goes to zero but once. However, considering the fact that the Zeta function is multivalued at the roots, any root could be used in the future of a Zero, and as such a product of all roots following the Hadamard product for the Zeta function can be used in the future of a Zero of the function.

$$
\zeta(s)=\mathrm{e}^{a+b s} \cdot s \cdot \prod_{\rho}\left(1-\frac{s}{\rho}\right) \mathrm{e}^{s / \rho} \cdot \prod_{n \geq 1}\left(1+\frac{s}{2 n}\right) \mathrm{e}^{-s / 2 n} \quad(\zeta(\rho)=0 \text { with } 0<\operatorname{Re}(\rho)<1)
$$

The Towering Zeta function $\zeta^{\vec{n}}(s)$ can in fact have an infinite number of convergence points in all its roots. 
All roots converge the function to the real line from the complex plane (Figure 5).

Starting from a given root, the future iterations of the Towering Zeta function function over successive arguments that start from a root will lead to a convergence for every root. Table 1 shows the iterations from a root $s$ in an upward future trend toward convergence to $C_{\infty}$. However starting from some past iteration, there exist an infinite number of roots that could be generated by a past iteration through zero.

Table 1 shows successive past values of arguments for the roots, that solve the iterated relation, $\zeta^{n+1}\left(z_{k}\right)=\zeta^{n}\left(z_{k}\right)$, where $n$ runs up the tables in a future direction, i.e. arguments of $\zeta^{\frac{1}{n+1}}\left(z_{k}\right)$ lead to $\zeta^{\vec{n}}\left(z_{k}\right)$. As can be seen from Table 1, the function can become almost oscillatory for some values of negative roots. Values down the table are arguments that can generate roots as starting arguments of successive values of $\zeta^{\vec{n}}\left(z_{k}\right)$.

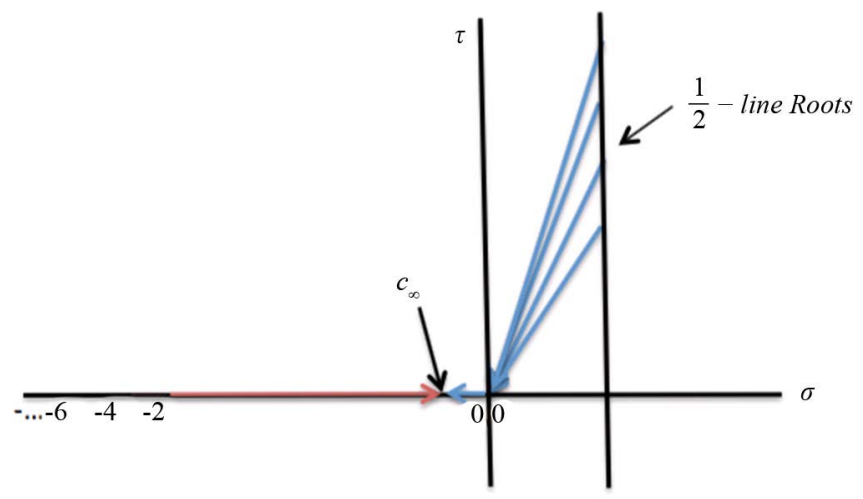

Figure 5. Shows the convergence from positive complex roots of the function to the real line.

Table 1. Iterations of the roots of the Towering Zeta Function over real arguments.

\begin{tabular}{ll}
\hline$\zeta^{n+1}(s)=\zeta\left(\zeta^{\tilde{n}}(s)\right)$ \\
\hline$\infty$ & $c_{\infty}$ \\
\hline$:$ & $:$ \\
\hline 17 & -0.29595806723778959429546880727279905000000000000000 \\
16 & -0.29580152831200018096290646344253482702467849073213 \\
15 & -0.29610685544242180860201784355977774292750241992426 \\
14 & -0.29551147448528153468605540107430168095507096662307 \\
13 & -0.29667304591820569539524370288435971088621053886270 \\
12 & -0.29440910387263694790663240676204680320270972628433 \\
11 & -0.29883017491388564173005702091107050051340373827686 \\
10 & -0.29022915571595152652543096855529715421054509417251 \\
9 & -0.30708636451194022129234803037380574262347512272602 \\
8 & -0.27451684815677210287939135693311577140783360600261 \\
7 & -0.33925706658308498350126705482505739973880105347080 \\
6 & 0.21728231379886310314230045114591184991388919709022 \\
5 & -0.47450768974007172623842641724875639785397472932299 \\
4 & -0.02860979985485943088252867867522696522057632451858 \\
3 & -1.4603545088095868128894991525148973697655036386338 \\
2 & $-1 / 2$ \\
1 & 0 \\
$0 \uparrow$ & $s$ \\
\hline
\end{tabular}


LEMMA 3: Le $t 0<r<\frac{1}{4}, s_{0}=\sigma_{0}+i \tau_{0}$, and let $f\left(s_{0}\right)=\zeta^{\vec{\infty}}\left(s_{0}\right)$, be a non-vanishing function on the disc $\left|s_{0}\right| \leq r$, which is analytic in the interior. Then,

$$
\max _{\left|s_{0}\right| \leq r}\left|\zeta\left(\frac{3}{4}\right)-c_{n}\right|<-\pi
$$

Use Voronin's theorem and let $f\left(s_{0}\right)=\zeta^{\vec{n}}\left(s_{0}\right)$. Then, $s_{0}=\zeta^{\overrightarrow{n-1}}\left(s_{0}\right)$

$$
\max _{\left|s_{0}\right| \leq r}\left|\zeta\left(\frac{3}{4}+\zeta^{\overline{n-1}}\left(s_{0}\right)+i t_{0}\right)-\zeta^{\vec{n}}\left(s_{0}\right)\right|<\varepsilon_{0} .
$$

Further, since $\frac{3}{4}+i t_{0} \neq 0$ if $t_{0} \in Z$, the constant $\varepsilon_{0}$ cannot be zero. However, we can choose $z_{0}$ and make

$$
\begin{aligned}
& \left(\frac{3}{4}+\zeta^{\overline{n-1}}\left(s_{0}\right)+i t_{0}\right)=\zeta^{\overline{n-1}}\left(z_{0}\right) \\
& \rightarrow \zeta^{\overline{n-1}}\left(z_{0}\right)-\zeta^{\overline{n-1}}\left(s_{0}\right)=\frac{3}{4}+i t_{0}
\end{aligned}
$$

So that $\max _{\left|s_{0}\right| \leq r}\left|\zeta^{\vec{n}}\left(z_{0}\right)-\zeta^{\vec{n}}\left(s_{0}\right)\right|<\varepsilon_{0}$. Obviously as $n \rightarrow \infty$, the convergence demands that $\varepsilon_{0} \rightarrow 0$.

As $n \rightarrow \infty, \varepsilon_{0} \rightarrow 0$. Now choose $\overline{\zeta^{n-1}}\left(s_{0}\right)=0$.

$$
\max _{\left|s_{0}\right| \leq r}\left|\zeta\left(\frac{3}{4}+i t_{0}\right)-c_{n}\right|<\varepsilon_{0} .
$$

Then there exists a negative constant such that

$$
\zeta^{\vec{n}}(0)=c_{n} \approx\left\{\begin{array}{cc}
-0.5000000000 & n=1 \\
-0.2078862250 & n=2 \\
-0.3448741787 & n=3 \\
\vdots & \vdots \\
-0.2954619778 & n=\infty
\end{array}\right.
$$

Then,

$$
\max _{|0| \leq r}\left|\zeta\left(\frac{3}{4}+i t_{n}\right)-c_{n}\right|<\varepsilon_{n}
$$

The constants $t_{0}$ must vanish at infinity since $\zeta^{\vec{n}}(0)$ is self similar and $\zeta^{\vec{\infty}}\left(c_{\infty}\right)=c_{\infty}$, and any approximations of a constant function must be a constant-function, then, one suspiciously finds that:

$$
\max _{\left|s_{0}\right|=0} \mid \zeta\left(\frac{3}{4}\right)-\left\{\begin{array} { c c } 
{ - 0 . 5 0 0 0 0 0 0 0 0 0 } & { n = 1 } \\
{ - 0 . 2 0 7 8 8 6 2 2 5 0 } & { n = 2 } \\
{ - 0 . 3 4 4 8 7 4 1 7 8 7 } & { n = 3 } \\
{ \vdots } & { \vdots } \\
{ - 0 . 2 9 5 4 6 1 9 7 7 8 } & { n = \infty }
\end{array} \approx \left\{\begin{array}{cc}
-2.941285387 \cdots, & n=1 \\
-3.233399162 \cdots, & n=2 \\
-3.096411208 \cdots, & n=3 \\
\vdots & \vdots \\
-3.145380381 \cdots, & n \rightarrow \infty
\end{array} \mid<\varepsilon_{0} .\right.\right.
$$

This value deviates from $\pi$ by $0.003787727780215700 \cdots$.

I will now discuss the relationship between the Towering Zeta function $\zeta^{\vec{n}}(z)$ and its derivatives. Differentiating $\zeta^{\vec{n}}(z)$, 


$$
\begin{gathered}
\frac{\mathrm{d}}{\mathrm{d} z} \zeta^{\vec{n}}(z)=\zeta\left(1, \zeta^{\overline{n-1}}(z)\right) \zeta\left(1, \zeta^{\overline{n-2}}(z)\right) \zeta\left(1, \zeta^{\overline{n-3}}(z)\right) \cdots \zeta(1, z)=\prod_{k=1}^{n-1}\left(\frac{\mathrm{d} \zeta^{\vec{k}}(z)}{\mathrm{d} z}\right) \\
\frac{\mathrm{d}}{\mathrm{d} z} \zeta^{\vec{n}}(z)=\prod_{k=0}^{n-1} \zeta\left(1, \zeta^{\vec{k}}(z)\right)
\end{gathered}
$$

Thus the derivative of the function $\zeta^{\vec{n}}(z)$ is just products of the derivatives of the iterate functions taken over $n-1$ values. Note that the derivatives of $\left(g^{\vec{n}}\right)$,

$$
\left(g^{\vec{n}}\right)^{\prime}(z)=\left([\log c]^{n}\right) \prod_{k=1}^{n-1} g^{\vec{k}}(z)
$$

have been shown by D.L. Shell [6], to be periodic. Obviously, the power towers are intimately related to the Towering Zeta function and the almost periodic relationship is exactly the sort of behavior one sees with the Zeta function:

$$
\begin{gathered}
\zeta\left(\left[\begin{array}{l}
0.95567279697780404255437895947517132067057510101417 \\
-30.000000006354409385604136689519632230386337754576 \\
-41.999999999999999867430211201873569223963122161146 \\
0.97651231492066129474435027600741771913283805368063 \\
-22.000360302508129001125278890966636148140124168019 \\
-21.985521871353759756816256045263338254747062798673 \\
-21.985532002522436978683430118473228296431588342576
\end{array}\right]\right) \\
\approx\left[\begin{array}{l}
-21.985532002522436978673430118473228296400065068011 \\
0.95567179697780404255436748814883558583102891315578 \\
-30.000000006354409385604136689521105193219591530893 \\
-41.999999999999999857430211201873569223963122331657 \\
0.97651231492066129474435027600741771913283810174678 \\
-22.000630302508129001125278890966636148140124543817 \\
-21.985521871353759756816256045263338254747062795690
\end{array}\right]
\end{gathered}
$$

\section{LEMMA 4:}

For $\mathfrak{R}\left(\zeta^{\overline{n-1}}(z)\right)>1, \frac{\mathrm{d}}{\mathrm{d} z}\left(\zeta^{\vec{n}}(z)\right)=\ln \left(\frac{v^{-\zeta^{\vec{n}}(z)}}{\prod_{u \neq v}^{\infty} v^{-u^{-\zeta^{n-1}}(z)}}\right)$.

Proof:

$$
\begin{gathered}
\zeta^{\prime}\left(\zeta^{\overline{n-1}}(z)\right)=\zeta\left(1, \zeta^{\overline{n-1}}(z)\right) \\
\zeta\left(1, \zeta^{\overline{n-1}}(z)\right)=\frac{\mathrm{d}}{\mathrm{d} z} \zeta\left(\zeta^{\overline{n-1}}(z)\right)=\frac{\mathrm{d}}{\mathrm{d} z}\left(\zeta^{\vec{n}}(z)\right)=-\sum_{v=2}^{\infty} \frac{\ln v}{v^{\zeta^{n-1}}(z)}
\end{gathered}
$$

Differentiating $\zeta^{\vec{n}}(z)$, one gets:

$$
\frac{\mathrm{d} \zeta^{\vec{n}}(z)}{\mathrm{d} z}=\prod_{k=1}^{n-1}\left(\frac{\mathrm{d} \zeta^{\vec{k}}(z)}{\mathrm{d} z}\right)
$$

Which is the same as the power tower derivatives when $c=e$.

$$
\left(g^{\vec{n}}\right)^{\prime}(z)=\left([\log C]^{n}\right) \prod_{k=1}^{n-1} g^{\vec{k}}(z)
$$


Hence,

$$
\frac{\mathrm{d} \zeta^{\vec{n}}(z)}{\mathrm{d} z}=\sum_{v=2}^{\infty} \ln v^{-v^{-\zeta^{n-1}}(z)}=\ln \prod_{v=2}^{\infty} v^{-v^{-\zeta^{n-1}}(z)}
$$

Noting that

$$
\zeta^{\vec{n}}(z)=\sum_{a_{n}=1 a_{n-1}=1}^{\infty} \prod_{n}^{-\prod_{a_{n-2}=1}^{\infty} a_{n-1}-\prod_{a_{n-3}=1}^{\infty} a_{n-2}-\cdots-\prod_{a_{1}=1}^{\infty} a_{2}-\prod_{a_{0}=1}^{\infty} a_{1}^{-a_{0}}{ }^{-z}}
$$

The derivative becomes,

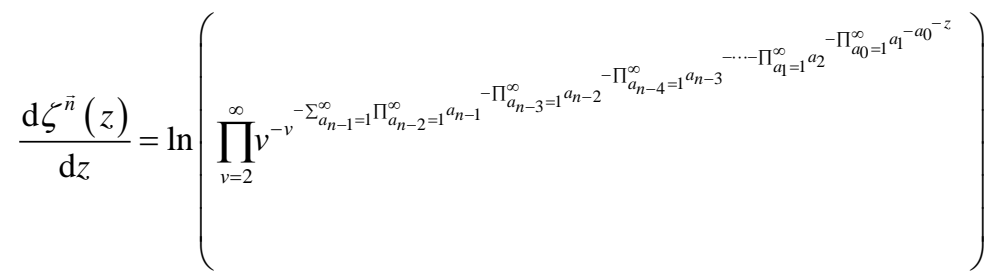

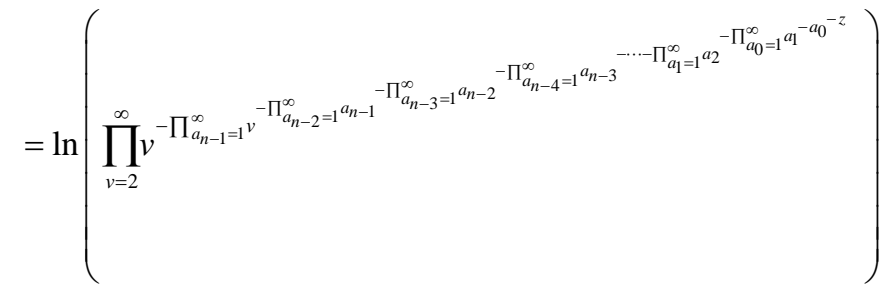

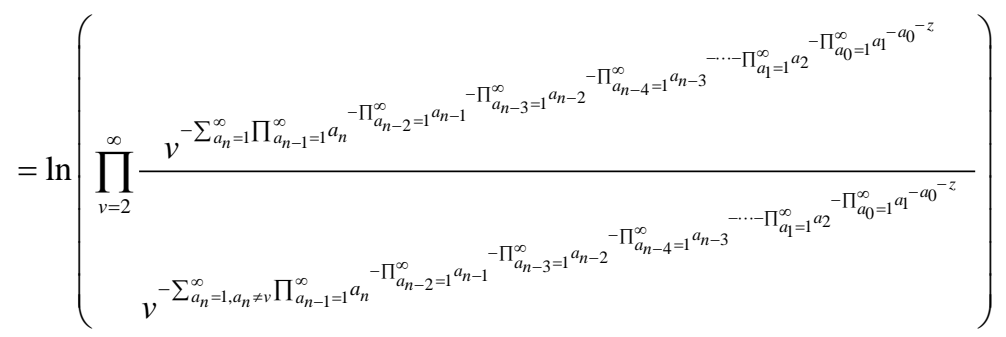

Put $a_{k+1} \rightarrow u$, and use

$$
\begin{aligned}
& \zeta^{\vec{n}}(z)=\sum_{a_{n}=1 a_{n-1}=1}^{\infty} \prod_{n}^{-\prod_{a_{n-2}=1}^{\infty} a_{n-1}-\prod_{a_{n-3}=1}^{\infty} a_{n-2}-\cdots-\prod_{a_{1}=1}^{\infty} a_{2}^{-}-\prod_{a_{0}=1}^{\infty} a^{a_{1}^{-a_{0}-z}}} \\
& \frac{\mathrm{d} \zeta^{\vec{n}}(z)}{\mathrm{d} z}=\ln \left(\prod_{v=2}^{\infty} \frac{v^{-\zeta^{\vec{n}}(z)}}{\prod_{u=1, u \neq v}^{\infty} v^{-u^{-\zeta^{n-1}(z)}}}\right)
\end{aligned}
$$

LEMMA5: Define $Z=\{s \in \mathbb{C} \mid \zeta(s)=0$, and $0 \leq \mathfrak{R}(s)>1\}$ and let $s \in \mathbb{C}$ and, $s=\sigma+i \tau$. Define $Z_{0}=\left\{s_{0} \in \mathbb{C} \mid \zeta\left(\zeta^{\overline{n-1}}\left(s_{0}\right)\right)=0\right.$ and $\left.0 \leq \mathfrak{R}\left(s_{0}\right)>1\right\}$ and let $s_{0} \in \mathbb{C}, n \in \mathbb{N}$, and $s_{0}=\sigma_{0}+i \tau_{0}$, then, $Z_{0} \subset\left\{s_{0} \in \mathbb{C} \mid\left\{\zeta^{\overline{n-1}}\left(s_{0}\right)=s\right\}, n \geq 1\right\}$.

Proof:

First we verify (102). 


$$
\begin{aligned}
& \frac{\mathrm{d} \zeta(s)}{\mathrm{d} z}=\ln \left(\prod_{v=1}^{\infty}\left(\frac{v^{-\zeta(s)}}{\prod_{u=1, u \neq v}^{\infty} v^{-u^{-s}}}\right)\right) \\
&= \ln \left(\prod_{v=1}^{\infty}\left(\prod_{u \neq v}^{\infty} v^{-\zeta(s)+u^{-s}}\right)\right) \\
&= \ln \left(\prod_{v=1}^{\infty} \frac{\prod_{u=1}^{\infty} v^{u^{-s}-\zeta(s)}}{v^{v^{-s}}}\right) \\
&= \ln \left(\prod_{v=1}^{\infty} \frac{v^{\sum_{u=1}^{\infty} u^{-s}-\zeta(s)}}{v^{v^{-s}}}\right) \\
&=\ln \left(\prod_{v=1}^{\infty} v^{-v^{-s}}\right)
\end{aligned}
$$

Consider the product formula:

$$
\frac{\mathrm{d} \zeta^{\vec{n}}(z)}{\mathrm{d} z}=\prod_{k=1}^{n-1}\left(\frac{\mathrm{d} \zeta^{\vec{k}}(z)}{\mathrm{d} z}\right)
$$

Let the root $s$ occur at the $m^{\text {th }}$ iteration of the product formula, at $k=m$. Then,

$$
\left.\frac{\mathrm{d}}{\mathrm{d} z} \zeta^{\vec{n}}(z)\right]_{\zeta^{\vec{m}}(z)=s}=\prod_{k=0}^{m-1}\left(\ln \prod_{v=1}^{\infty} v^{-v^{-\zeta^{\vec{k}}\left(s_{0}\right)}}\right) \ln \left(\prod_{v=1}^{\infty} \frac{1}{\prod_{u \neq v}^{\infty} v^{-u^{-s}}}\right) \prod_{k=m+1}^{n-1}\left(\ln \prod_{v=1}^{\infty} v^{-v^{-\zeta^{-\vec{k}}\left(s_{0}\right)}}\right)
$$

This separates the products into three terms, the first term, $P$, being the iterates before the root $s$ is encountered, $P \in k=0, \cdots, m-1$, and the second term $N$, being the iterate that produces a root at $N \in k=m$, and third term being the iterations after the root at $F \in k=m+1, \cdots, n-1$.

$$
P=\prod_{k=0}^{m-1}\left(\ln \prod_{v=1}^{\infty} v^{-v^{-\zeta^{\vec{k}}\left(s_{0}\right)}}\right), N=\ln \left(\prod_{v=1}^{\infty} \frac{1}{\prod_{u \neq v}^{\infty} v^{-u^{-s}}}\right), F=\prod_{k=m+1}^{n-1}\left(\ln \prod_{v=1}^{\infty} \frac{v^{-\zeta^{\overline{k+1}}\left(s_{0}\right)}}{\prod_{u \neq v}^{\infty} v^{-u^{-\zeta^{\vec{k}}}\left(s_{0}\right)}}\right)
$$

One has to determine if the factors $P, N, F$, can vanish in a given range of values of the arguments, $s$. Before determining these products, the following Lemmas are necessary.

LEMMA 6: If $\zeta(s)=0$, and if $p, q, m, n \in \mathrm{Z}$, and if $0<p<q<m<n$, and $\zeta^{\vec{p}}(z)=\zeta^{\vec{q}}(z)$, then, $\zeta^{\vec{m}}(z) \neq S$.

Proof: Suppose there exists $\zeta^{\vec{p}}(z)=\zeta^{\vec{q}}(z)$. Then, iterations from $p$ to $q$ will be periodic and will only generate cyclic arguments when $\zeta^{p+k}(z)=\zeta^{\vec{q}}(z)=\zeta^{\vec{p}}(z)$, where, $k=q-p$. Thus the past iterations for $i<q$, will be stuck in an eternal loop and never generate future roots, $s$. This is true for both real and complex arguments, $z$ and for real and complex roots $s$.

LEMMA7: Let $\zeta^{\bar{m}}(z)=s$, then, if $\zeta(s)=0$, and if $\{p, q, m, k\} \in \mathrm{Z}$, and $0<p<q<m$, and $z \in \mathbb{C}$, and if for any $p, q, \quad \zeta^{\bar{p}}(z) \neq \zeta^{\bar{q}}(z)$, then, $P$ and $F$ are infinite power towers.

Proof:

If $p, q, m, n \in \mathrm{Z}$, and $0<p<q<m<n$, and if for any $p$ or $q, \quad \zeta^{\bar{p}}(z) \neq \zeta^{\bar{q}}(z)$ then the sequence of iterations of the zeta function $\zeta^{\dot{m}}(z)=s$ has no past purely periodic arguments and as such there can be no other repelling, neutral or super-attracting points until the root itself is reached.

Then by induction, there are no past periodic arguments in the factor $P$ prior to encountering this root. There must exists an infinite past for the iterations leading to root $s$. Then, the only critical point is the root itself and the sum of all such points will be the root. The root could be written as an infinite power tower of $s_{m}=\sum g^{\vec{\infty}}(z)$. 
The same argument leads $F$ to an infinite power tower.

Noting that

$$
\begin{aligned}
& \zeta^{\vec{m}}\left(s_{0}\right)=s \\
& \zeta^{\overrightarrow{m+1}}\left(s_{0}\right)=\zeta\left(\zeta^{\vec{m}}\left(s_{0}\right)\right)=c_{1}=0 \\
& \zeta^{\overline{m+2}}\left(s_{0}\right)=\zeta(0)=c_{2}=-\frac{1}{2}, \\
& \zeta^{\overline{m+3}}\left(s_{0}\right)=\zeta\left(-\frac{1}{2}\right)=c_{3}=-0.2078862250 \cdots, \\
& \zeta^{\overline{m+4}}\left(s_{0}\right)=\zeta(-0.2078862250)=c_{4}=-0.3448741787 \cdots, \\
& \zeta^{\vec{\infty}}\left(s_{0}\right)=s_{\infty}=-0.2959050806 \cdots, \\
& F=\prod_{k=m+1}^{\infty}\left(\ln \prod_{v=1}^{\infty} \frac{v^{-\zeta^{\overline{k+1}}\left(s_{0}\right)}}{\prod_{u \neq v}^{\infty} v^{-u^{-\zeta^{k}}\left(s_{0}\right)}}\right) \\
& =\left(\sum_{v=1}^{\infty} \ln \left(\frac{v^{-\left(-\frac{1}{2}\right)}}{\prod_{u \neq v}^{\infty} v^{-u^{-0}}}\right) \sum_{v=1}^{\infty} \ln \left(\frac{v^{-(-0.2078862250)}}{\prod_{u \neq v}^{\infty} v^{-u^{-\left(-\frac{1}{2}\right)}}}\right) \sum_{v=1}^{\infty} \ln \left(\frac{v^{-(-0.3448741787)}}{\prod_{u \neq v}^{\infty} v^{-u^{-(-0.2078862250)}}}\right) \cdots \sum_{v=1}^{\infty} \ln \left(\frac{v^{-c_{\infty}}}{\prod_{u \neq v}^{\infty} v^{-u^{-\left(c_{\infty}\right)}}}\right)\right) \\
& =\prod_{k=0}^{\infty} \sum_{v=1}^{\infty} \ln \left(\frac{v^{-\left(c_{k}\right)}}{v^{-\sum_{u \neq v}^{\infty} u^{-\left(c_{k-1}\right)}}}\right)
\end{aligned}
$$

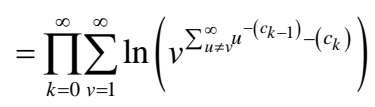

$$
\begin{aligned}
& =\prod_{k=0}^{\infty}\left(\sum_{v=1}^{\infty}\left(\left(\left(\sum_{u \neq v}^{\infty} u^{-\left(c_{k-1}\right)} \ln (v)\right)-\left(c_{k}\right) \ln (v)\right)\right)\right) \\
& =\prod_{k=0}^{\infty}\left(\sum_{v=1}^{\infty}\left(\sum_{u=1}^{\infty}\left\{u^{-\left(c_{k-1}\right)} \ln (u)-v^{-\left(c_{k-1}\right)} \ln (v)\right\}-\left\{\left(c_{k}\right) \ln (v)\right\}\right)\right)=0
\end{aligned}
$$$$
\text { Thus } F=0 \text {. }
$$

The factor $N$ is given by:

$$
\begin{gathered}
N=\ln \left(\prod_{v=1}^{\infty} \frac{1}{\prod_{u \neq v}^{\infty} v^{-u^{-s}}}\right)=\sum_{v=1}^{\infty} \ln \left(\prod_{u \neq v}^{\infty}\left(v^{u^{-s}}\right)\right) \\
=\sum_{v=1}^{\infty}\left(-\sum_{u=1}^{\infty}\left(\frac{\ln (u)}{u^{s}}\right)+\frac{\ln (v)}{v^{s}}\right) \\
=(\zeta(1, s)-\zeta(1, s))=0 .
\end{gathered}
$$

Both the above factors are zero, hence the proof. It is obvious that if a root occurs in any of the arguments of the function iterates of $\zeta^{\vec{\infty}}(z)$, then, the derivatives of $\zeta^{\vec{\infty}}(z)$ must vanish since it converges to a constant. Now the conditions that allow a root to be encountered depend on $P$.

It is worth noting that the function $\zeta^{\vec{n}}(z)$, and the arguments $\zeta^{\overline{n-1}}(z)$, are discontinuous functions over the complex plane. In other words they jump in values over each iteration and may never hit a root for some values 
of $z$.

Let $z_{0}$ be a starting value that does not hit a root.

The relationship given by the Hadamard factorization thus represents the relationship between the iterated functions $\zeta^{\vec{n}}\left(z_{0}\right)$, and roots $\rho$ of the zeta function.

$$
\zeta^{\vec{n}}\left(z_{0}\right)=\frac{\pi^{\frac{\zeta^{n-1}\left(z_{0}\right)}{2}} \prod_{\rho}^{\infty}\left(1-\frac{\zeta^{\overline{n-1}}\left(z_{0}\right)}{\rho}\right)}{2\left(\zeta^{\overline{n-1}}\left(z_{0}\right)-1\right) \Gamma\left(\frac{\zeta^{\overline{n-1}}\left(z_{0}\right)}{2}+1\right)}=\zeta^{\overline{n-1}}\left(z_{0}\right) \int_{0}^{\infty}\left(\frac{x-x}{x^{\zeta^{n-1}}\left(z_{0}\right)+1}\right) d x
$$

Obviously, the argument $\zeta^{\overline{n-1}}\left(z_{0}\right)=1$, is a pole of the function and so we assume $\zeta^{\overline{n-1}}\left(z_{0}\right)<1$. From the reflection formula,

$$
\pi^{-\frac{\xi^{n-1}\left(z_{0}\right)}{2}} \zeta^{\vec{n}}\left(z_{0}\right) \Gamma\left(\frac{\zeta^{\overline{n-1}}\left(z_{0}\right)}{2}\right)=\pi^{-\frac{1-\zeta^{n-1}\left(z_{0}\right)}{2}} \zeta\left(1-\zeta^{\overline{n-1}}\left(z_{0}\right)\right) \Gamma\left(\frac{1}{2}-\frac{\zeta^{\overline{n-1}}\left(z_{0}\right)}{2}\right)
$$

Since $\zeta^{\overrightarrow{n-1}}\left(z_{0}\right)$ never hits a root, we can divide across by the reflection function,

$$
\begin{gathered}
\left(\frac{\pi^{\frac{\zeta^{n-1}\left(z_{0}\right)}{2}} \prod_{\rho}^{\infty}\left(1-\frac{\zeta^{\overline{n-1}}\left(z_{0}\right)}{\rho}\right)}{2\left(\zeta^{\overline{n-1}}\left(z_{0}\right)-1\right) \Gamma\left(\frac{\zeta^{\overline{n-1}}\left(z_{0}\right)}{2}+1\right)}\right)\left(\frac{\pi^{-\frac{\zeta^{n-1}}{2}\left(z_{0}\right)} \zeta^{\vec{n}}\left(z_{0}\right) \Gamma\left(\frac{\zeta^{\overline{n-1}}\left(z_{0}\right)}{2}\right)}{\pi^{-\frac{1-\zeta^{n-1}}{2}\left(z_{0}\right)} \zeta\left(1-\zeta^{\overline{n-1}}\left(z_{0}\right)\right) \Gamma\left(\frac{1}{2}-\frac{\zeta^{\overline{n-1}}\left(z_{0}\right)}{2}\right)}\right)=\zeta^{\vec{n}}\left(z_{0}\right) \\
\left(\frac{\prod_{\rho}^{\infty}\left(1-\frac{\zeta^{\overline{n-1}}\left(z_{0}\right)}{\rho}\right) \zeta^{\vec{n}}\left(z_{0}\right) \Gamma\left(\frac{\zeta^{\overline{n-1}}\left(z_{0}\right)}{2}\right)}{2\left(\zeta^{\overline{n-1}}\left(z_{0}\right)-1\right) \pi^{\frac{\zeta^{n-1}}{2}\left(z_{0}\right)} \zeta\left(1-\zeta^{\overline{n-1}}\left(z_{0}\right)\right)}\right)\left(\frac{\sqrt{\pi}}{\Gamma\left(\frac{\zeta^{\overline{n-1}}\left(z_{0}\right)}{2}+1\right) \Gamma\left(\frac{1}{2}-\frac{\zeta^{\overline{n-1}}\left(z_{0}\right)}{2}\right)}\right)=\zeta^{\vec{n}}\left(z_{0}\right)
\end{gathered}
$$

Since $\zeta^{\vec{n}}\left(z_{0}\right) \neq 0$, one can divide across by $\zeta^{\vec{n}}\left(z_{0}\right)$,

$$
\left(\frac{\prod_{\rho}^{\infty}\left(1-\frac{\zeta^{\overline{n-1}}\left(z_{0}\right)}{\rho}\right) \Gamma\left(\frac{\zeta^{\overline{n-1}}\left(z_{0}\right)}{2}\right)}{2\left(\zeta^{\overline{n-1}}\left(z_{0}\right)-1\right) \pi^{\frac{\zeta^{n-1}\left(z_{0}\right)}{2}} \zeta\left(1-\zeta^{\overline{n-1}}\left(z_{0}\right)\right)}\right)\left(\frac{\sqrt{\pi}}{\Gamma\left(\frac{\zeta^{\overline{n-1}}\left(z_{0}\right)}{2}+1\right) \Gamma\left(\frac{1}{2}-\frac{\zeta^{\overline{n-1}}\left(z_{0}\right)}{2}\right)}\right)=1
$$

Using

$$
\begin{gathered}
\frac{\sqrt{\pi}}{\Gamma\left(\frac{\zeta^{\overline{n-1}}(z)}{2}+1\right) \Gamma\left(\frac{1}{2}-\frac{\zeta^{\overline{n-1}}(z)}{2}\right)}=\prod_{k=1}^{\infty}\left(1-\frac{\zeta^{\overline{n-1}}(z)}{2 k-1}\right)\left(1+\frac{\zeta^{\overline{n-1}}(z)}{2 k}\right) \\
\left(\frac{\prod_{\rho}^{\infty}\left(1-\frac{\zeta^{\overline{n-1}}\left(z_{0}\right)}{\rho}\right) \Gamma\left(\frac{\zeta^{\overline{n-1}}\left(z_{0}\right)}{2}\right)}{2\left(\zeta^{\overline{n-1}}\left(z_{0}\right)-1\right) \zeta\left(1-\zeta^{\overline{n-1}}\left(z_{0}\right)\right)}\right)\left(\pi^{\frac{\zeta^{\frac{n-1}{2}}\left(z_{0}\right)}{2}} \frac{\sqrt{\pi}}{\Gamma\left(\frac{\zeta^{n-1}(z)}{2}+1\right) \Gamma\left(\frac{1}{2}-\frac{\zeta^{n-1}(z)}{2}\right)}\right)=1
\end{gathered}
$$




$$
\begin{gathered}
\zeta\left(1-\zeta^{\overline{n-1}}\left(z_{0}\right)\right)=\left(\frac{\pi^{\frac{\zeta^{n-1}}{2}\left(z_{0}\right)} \prod_{\rho}^{\infty}\left(1-\frac{\zeta^{\overline{n-1}}\left(z_{0}\right)}{\rho}\right) \sqrt{\pi} \Gamma\left(\frac{\zeta^{\overline{n-1}}\left(z_{0}\right)}{2}\right)}{2\left(\zeta^{\overline{n-1}}\left(z_{0}\right)-1\right) \Gamma\left(\frac{\zeta^{\overline{n-1}}(z)}{2}+1\right) \Gamma\left(\frac{1}{2}-\frac{\zeta^{\overline{n-1}}(z)}{2}\right)}\right) \\
\zeta^{\bar{n}}\left(z_{0}\right)=\frac{\pi^{\left.\frac{\zeta^{n-1}}{2} z_{0}\right)} \prod_{\rho}^{\infty}\left(1-\frac{\zeta^{\overline{n-1}}\left(z_{0}\right)}{\rho}\right)}{2\left(\zeta^{\overline{n-1}}\left(z_{0}\right)-1\right) \Gamma\left(\frac{\zeta^{\overline{n-1}}\left(z_{0}\right)}{2}+1\right)} \\
\zeta\left(1-\zeta^{\overline{n-1}}\left(z_{0}\right)\right) \Gamma\left(\frac{1}{2}-\frac{\zeta^{\overline{n-1}}\left(z_{0}\right)}{2}\right)=\sqrt{\pi} \Gamma\left(\frac{\zeta^{\overline{n-1}}\left(z_{0}\right)}{2}\right) \zeta^{\vec{n}}\left(z_{0}\right)
\end{gathered}
$$

From the reflection formula,

$$
\zeta\left(1-\zeta^{\overline{n-1}}\left(z_{0}\right)\right) \Gamma\left(\frac{1}{2}-\frac{\zeta^{\overline{n-1}}\left(z_{0}\right)}{2}\right)=\pi^{\frac{1}{2}-\zeta^{\overline{n-1}}\left(z_{0}\right)} \Gamma\left(\frac{\zeta^{\overline{n-1}}\left(z_{0}\right)}{2}\right) \zeta^{\vec{n}}\left(z_{0}\right)
$$

From the power of $\pi$, the condition for no roots to be obtained during iterations is that $\zeta^{\overline{n-1}}\left(z_{0}\right)=0, \zeta^{\vec{n}}\left(z_{0}\right)=-\frac{1}{2}$.

$$
-\frac{1}{2}=\frac{\prod_{\rho}^{\infty}(1)}{2(-1) \Gamma(1)}=-\frac{1}{2}
$$

The condition $\zeta^{\overline{n-1}}\left(z_{0}\right)=0$ implies that a root $\zeta^{\overline{n-2}}\left(z_{0}\right)=\rho$ is a hit for arguments before $(n-1)^{\text {th }}$ iteration. Thus the condition demonstrates that any prior argument that is a root $\overline{\zeta^{n-2}}\left(z_{0}\right)=\rho$ will never have iterations that give another root again.

$$
\zeta^{\overrightarrow{n-1}}\left(z_{0}\right)=0, \zeta^{\vec{n}}\left(z_{0}\right)=-\frac{1}{2}
$$

Further, for $\mathfrak{R}(s)>1$, the iterates that lead to a root give

$$
\begin{gathered}
P=\prod_{k=0}^{m-1} \sum_{v=1}^{\infty} \ln \left(\frac{v^{-\zeta^{\overline{k+1}}(z)}}{\left.v^{-\sum_{u \neq u^{-}}^{\infty} \zeta^{-\zeta^{\vec{k}}(z)}}\right)}\right) \\
\left.=\prod_{k=0}^{m-1} \sum_{v=1}^{\infty} \ln \left(v^{\sum_{u \neq u^{-}}^{\infty}-\left(\zeta^{\vec{k}}(z)\right)}\right)-\left(\zeta^{\overline{k+1}}(z)\right)\right) \\
=\prod_{k=0}^{m-1}\left(\sum_{v=1}^{\infty}\left(\sum_{u \neq v}^{\infty}\left(u^{-\left(\zeta^{\vec{k}}(z)\right)} \ln (v)\right)-\left(\zeta^{\overline{k+1}}(z)\right) \ln (v)\right)\right) \\
=\prod_{k=0}^{m-1}\left(\sum_{v=1}^{\infty}\left(\sum_{u=1}^{\infty}\left\{u^{-\left(\zeta^{\vec{k}}\right)} \ln (u)-v^{-\left(\zeta^{\vec{k}}\right)} \ln (v)\right\}-\left\{\left(\zeta^{\overline{k+1}}\right) \ln (v)\right\}\right)\right)
\end{gathered}
$$




$$
=\prod_{k=0}^{m-1}\left(\sum_{v=2}^{\infty}\left(-\zeta^{\overline{k+1}}(z) \ln \zeta^{\overline{k+1}}(z) \ln (v)\right)\right)
$$

From, (124), one sees that the derivative vanishes at the root of given by some iterate $\zeta^{\overline{k+1}}(z)=0$. The condition that the derivative does not vanish when $0<\mathfrak{R}(s)=\mathfrak{R}\left(\zeta^{\vec{n}}(z)\right)<\frac{1}{2}$, leads to a solution of the Riemann Hypothesis. However, for the case $\mathfrak{R}(s) \geq 1$, when $\zeta^{\vec{n}}(z)$ is a root, none of the iterates vanish, since the iterates lead to the final root.

\section{Connection of the Towering Zeta Function to Exponential Power Towers}

To illustrate the convergence of the Towering Zeta function for $1>\mathfrak{R}(s)>0$, we start with:

$$
Z_{n}=(-1)^{a_{n}} 2^{b_{n}} \prod_{b_{n-1}=a_{n-1}=1}^{\infty} \prod^{\infty}\left(2^{b_{n}} a_{n}\right)^{\left(-Z_{n-1}\right)}
$$

Then,

$$
\begin{gathered}
\zeta^{\vec{n}}(z)=\sum_{a_{n}=1 b_{n}=1}^{\infty} Z_{n} \\
\text { Now use } \pi^{-\frac{s}{2}} \zeta(s) \Gamma\left(\frac{s}{2}\right)=\pi^{-\frac{1-s}{2}} \zeta(1-s) \Gamma\left(\frac{1-s}{2}\right) \\
\zeta\left(1-\zeta^{\overline{n-1}}(z)\right)=2^{\left(1-\zeta^{n-1}(z)\right)} \pi^{-\zeta^{n-1}(z)} \cos \left(\frac{\pi \zeta^{\overline{n-1}}(z)}{2}\right) \Gamma\left(\zeta^{\overline{n-1}}(z)\right) \zeta^{\vec{n}}(z)
\end{gathered}
$$

To see that the reflection formula obeys the power tower representation, take,

$$
\begin{aligned}
\zeta(1 & \left.-\zeta^{\overline{n-1}}(z)\right)=\sum_{a_{n}=1 b_{n}=1}^{\infty}\left((-1)^{a_{n}} 2^{b_{n}}\left(2^{b_{n}} a_{n}\right)^{\left(-1+\zeta^{n-1}(z)\right)}\right) \\
& =\sum_{a_{n}=b_{n}=1}^{\infty} \sum^{\infty}\left(\frac{1}{2^{b_{n}} a_{n}}(-1)^{a_{n}} 2^{b_{n}}\left(2^{b_{n}} a_{n}\right)^{\left(\zeta^{n-1}(z)\right)}\right) \\
& =\sum_{a_{n}=1 b_{n}=1}^{\infty} \sum^{\infty}\left(\frac{1}{a_{n}}(-1)^{a_{n}} \prod_{b_{n-1}=a_{n-1}=1}^{\infty} \prod^{\infty}\left(2^{b_{n}} a_{n}\right)^{\left(z_{n-1}\right)}\right)
\end{aligned}
$$

Then, since $Z_{n}=(-1)^{a_{n}} 2^{b_{n}} \prod_{b_{n-1}=1}^{\infty} \prod_{a_{n-1}=1}^{\infty}\left(2^{b_{n}} a_{n}\right)^{\left(-Z_{n-1}\right)}$, the two zeta functions can be written in the form:

$$
\left.\begin{array}{l}
\zeta\left(1-\zeta^{\overline{n-1}}(z)\right)=\sum_{a_{n}=1 b_{n}=1}^{\infty} \sum^{\infty}\left(\frac{(-1)^{a_{n}} \prod_{b_{n-1}=1}^{\infty} \prod_{a_{n-1}=1}^{\infty}\left(2^{b_{n}} a_{n}\right)^{\left(Z_{n-1}\right)}}{a_{n}}\right) \\
\zeta^{\vec{n}}(z)=\sum_{a_{n}=1 b_{n}=1}^{\infty} \sum^{\infty}\left(2^{b_{n}}(-1)^{a_{n}} \prod_{b_{n-1}=1 a_{n-1}=1}^{\infty} \prod^{\infty}\left(2^{b_{n}} a_{n}\right)^{\left(-z_{n-1}\right)}\right)
\end{array}\right\}
$$

LEMMA 8: The fixed points of the Towering Zeta function are rational functions of its roots.

\section{Proof:}

Putting

$$
\left.\begin{array}{l}
\varnothing_{z}(n)=2^{b_{n}}(-1)^{a_{n}} \prod_{b_{n-1}=1 a_{n-1}=1}^{\infty}\left(2^{b_{n}} a_{n}\right)^{-2^{b_{n-1}} a_{n-1} \psi_{n-1}} \\
\psi_{z}(n)=\frac{1}{a_{n}}(-1)^{a_{n}} \prod_{b_{n-1}=1 a_{n-1}=1}^{\infty} \prod^{\infty}\left(2^{b_{n}} a_{n}\right)^{-\varnothing_{n-1}}
\end{array}\right\}
$$


Thus,

$$
\left.\begin{array}{l}
\zeta\left(1-\zeta^{\overline{n-1}}(z)\right)=\sum_{a_{n}=1 b_{n}=1}^{\infty}\left(\psi_{z}(n)\right) \\
\left.\zeta^{\vec{n}}(z)=\sum_{a_{n}=1 b_{n}=1}^{\infty} \sum_{z}(n)\right)
\end{array}\right\}
$$

And so,

$$
\begin{gathered}
\left(a_{n} \psi_{z}(n)\right)^{-\frac{1}{\varnothing_{z}(n-1)}}=\left(\frac{\varnothing_{z}(n)}{2^{b_{n}}}\right)^{-\frac{1}{2^{b_{n-1}} a_{n-1} \psi_{z}(n-1)}} \\
2^{b_{n-1} a_{n-1} \psi_{z}(n-1)} \sqrt{a_{n} \psi_{z}(n)}=\sqrt[\varnothing_{z}(n-1)]{\frac{\varnothing_{z}(n)}{2^{b_{n}}}}
\end{gathered}
$$

The fixed points are obtained by the solutions to the relation:

$$
\sqrt[a_{n} \psi_{z}(n)]{a_{n} \psi_{z}(n)}=\sqrt[\frac{\varnothing_{z}(n)}{2^{b_{n}}}]{\frac{\varnothing_{z}(n)}{2^{b_{n}}}}
$$

This can be simplified by putting $\frac{\varnothing_{z}(n)}{2^{b_{n}}}=\varphi_{z}(n), a_{n} \psi_{z}(n)=\phi_{z}(n)$.

$$
\sqrt[\varphi_{z}(n)]{\varphi_{z}(n)}=\sqrt[\phi_{z}(n)]{\phi_{z}(n)}
$$

Power towers of the form (145) represent rational functions.

Remark 1. The only real algebraic solutions to (137) are 1, 2, and 4, since as shown in (23), functions of the power form (137) have an equivalence for the values 2 , and 4 :

$$
\sqrt{2}^{\sqrt{2}^{\sqrt{2}^{\sqrt{2}}}}=\sqrt{4}^{\sqrt{4}^{\sqrt{4}^{\sqrt{4}}}}=2
$$

It is easy to see that if $\varphi_{z}(n) \neq \phi_{z}(n)$ then the unique divisors of $\varphi_{z}(n)$ and $\phi_{z}(n)$ are the same. It has been shown that values for $\varphi_{z}(n) \neq \phi_{z}(n)$ are transcendental number.

For real power towers, the prime divisors $z_{k}^{\alpha_{k}}$ of $\phi_{z}(n)$ and the prime divisors $z_{k}^{\beta_{k}}$ of $\varphi_{z}(n)$ are the same. Thus for the complex values, if there is an infinite number of unique divisors, then,

$$
\left.\begin{array}{l}
\phi_{z}(n)=\prod_{k=1}^{\infty} z_{k}^{\alpha_{k}} \\
\varphi_{z}(n)=\prod_{k=1}^{\infty} z_{k}^{\beta_{k}}
\end{array}\right\}
$$

$\varphi_{n}$ must be divisible by $\phi_{n}$ leaving a factors that are the roots of unity. To see that the remaining factors are the roots of unity take $\varphi_{z}(n)=f(z) \phi_{z}(n)$, and so,

$$
\left(f(z) \phi_{z}(n)\right)^{\varphi_{z}(n)}=\left(\varphi_{z}(n)\right)^{f(z) \phi_{z}(n)}
$$

and from this

$$
\phi_{z}(n)=f(z)^{\frac{1}{f(z)-1}}, \varphi_{z}(n)=f(z)^{\frac{f(z)}{f(z)-1}}
$$

Write the rational function $\frac{1}{f(z)-1}$ as a rational function $\frac{1}{f(z)-1}=-s \in \mathbb{C}$. 
Then, $f(z)=\frac{s-1}{s}$, and,

$$
\varphi_{z}(n)=\left(\frac{s}{s-1}\right)^{s}, \phi_{z}(n)=\left(\frac{s}{s-1}\right)^{1-s}
$$

Thus the reflection formula applies to the power tower representation:

$$
s \rightarrow 1-s, \varphi_{z}(n) \rightarrow \frac{1}{\phi_{z}(n)}=\left(\frac{s-1}{s}\right)^{1-s}, \phi_{z}(n)=\frac{1}{\varphi_{z}(n)} \rightarrow\left(\frac{s-1}{s}\right)^{s}
$$

The reflection formula then tells us that $\phi_{z}(n)$ and $\varphi_{z}(n)$ obey reciprocal relations. In Ref. [5] Shkliarski D., N. Chentzov, and I. Yaglom, show that for real integer values of $s$, the forms of equation (142) represent Power Towers that converge to real rational values.

DEFINITION 8. Define the set

$$
w_{n}=\phi_{z}(n)^{\vec{n}}(1)=\phi \circ \phi \circ \cdots \circ \phi(1), n=1,2, \cdots
$$

where $\phi^{\vec{n}}$ denotes the $n^{\text {th }}$ iterate of the map $\phi$ and converges.

$w_{n}$ converges with limit $\lambda \in \mathbb{C}$, then $\phi(\lambda)=\phi^{\lambda}=\lambda$. Thus,

$$
\phi_{z}(n)=\mathrm{e}^{\frac{2 i n \theta}{\mathrm{e}^{2 i n} \theta}}
$$

and so on with each such relation representing an entire function of $\phi_{z}(n)$.

LEMMA 9: (Shell: Ref. [7]). Let $f(x)=\mathrm{e}^{\frac{x}{\mathrm{e}^{x}}}, x \in \mathbb{C}$, then $\left\{f^{\vec{n}}(x)\right\}_{n \in \mathbb{N}}$ converges to $\mathrm{e}^{x}$, in some neighborhood of $\mathrm{e}^{x}$, if $|x|<1$ and can do so only if $|x|<1$.

Proof: Take the principal branch $k=0$.

$$
f^{\vec{n}}(x)=\frac{W\left(-\log \left(\mathrm{e}^{\frac{x}{\mathrm{e}^{x}}}\right)\right)}{-\log \left(\mathrm{e}^{\frac{x}{\mathrm{e}^{x}}}\right)}=\frac{W\left(-x \mathrm{e}^{-x}\right)}{-x \mathrm{e}^{-x}}=\frac{-x}{-x \mathrm{e}^{-x}}=\mathrm{e}^{x} .
$$

An even stronger condition can be placed on the convergence of the sequence (143) by Thron in Ref. [8].

LEMMA 10: (Thron; Ref. [8]):

If $a=\frac{x}{\mathrm{e}^{x}},|x|<1$, or if $x$ is a root of unity, then the sequence (143) converges to $\mathrm{e}^{x}$. For almost all $x$ such that $|x|=1$, the sequence diverges.

Galidakis Ref. [9] noted that the fixed points $h(c)$ of the functions $\phi_{z}(n)^{\vec{n}}$ are rationally indifferent or parabolic and that their multiplier is exactly $z=\mathrm{e}^{2 \pi \theta i}$. I.N. Baker and P.J. Rippon Ref. [10] showed that if $\theta$ is a centrum number, in particular if $\theta$ is non-Liouville number, then (143) diverges. The $n^{\text {th }}$ order power tower only converges to $n$ fixed-points on the circle when $\zeta^{\vec{n}}(x) \in s$, a root of the function and when

$x \in \mathrm{e}^{\frac{2 \pi n}{k} i}=\mathrm{e}^{2 n \theta i}$, with $\theta$ being a rational function.

Following Titshmarch, Ref. [11],

Let $\phi(x)$ be any function with a continuous derivative in the interval $\{a, b\}$. Then, if $\lfloor x\rfloor$ denotes the greatest integer not exceeding $x$,

$$
\sum_{a<n \leq b} \phi(n)=\int_{a}^{b} \phi(x) \mathrm{d} x+\int_{a}^{b}\left(\lfloor x\rfloor-x-\frac{1}{2}\right) \phi^{\prime}(x) \mathrm{d} x-\left(b-\lfloor b\rfloor-\frac{1}{2}\right) \phi(b)+\left(a-\lfloor a\rfloor-\frac{1}{2}\right) \phi(a)
$$

Following Titshmarch Ref. [11] page 14, taking $\phi(x)=x^{-s}, s \neq 1, a=1, b \rightarrow \infty$, one arrives at the well known 
Zeta relation:

$$
\zeta(s)=s \int_{1}^{\infty}\left(\frac{\lfloor x\rfloor-x+\frac{1}{2}}{x^{s+1}}\right) \mathrm{d} x+\frac{1}{s-1}+\frac{1}{2}, \mathfrak{R}(s)>1
$$

Since $\lfloor x\rfloor-x+\frac{1}{2}$ is bounded, (148) is convergent for $\mathfrak{R}(s)>0$.

For the region $0<\mathfrak{R}(s)<1$,

$$
\frac{s}{2} \int_{1}^{\infty}\left(\frac{1}{x^{s+1}}\right)=\frac{1}{2}
$$

and so

$$
\zeta(s)=s \int_{1}^{\infty}\left(\frac{\lfloor x\rfloor-x}{x^{s+1}}\right) \mathrm{d} x+\frac{s}{s-1}, \quad 0<\mathfrak{R}(s)<1
$$

Assuming the Riemann Hypothesis, the Towering Zeta Function follows this relation before convergence, i.e. when the real part of the arguments of the Zeta function is in the range $0<\mathfrak{R}(s)<1$.

Let $s$ be a root of the Zeta function. Assuming the Riemann hypothesis, when the Towering Zeta function converges, the next argument is of the form

$$
\zeta(\zeta(s))=-\frac{1}{2},\{-1<\mathfrak{R}(s)<0\}
$$

Thus, for some finite iterations of a starting argument, $z$, let $\zeta^{\vec{n}}(z)=s$ be a root of the Zeta function. Then, the inverse gives, $z=\zeta^{\bar{n}}(s)$ The Zeta function now follows the relation,

$$
\zeta^{\vec{\infty}}(0)=\zeta^{\vec{\infty}}(0) \int_{0}^{\infty}\left(\frac{\lfloor x\rfloor-x+\frac{1}{2}}{x^{\zeta^{\vec{\infty}}(0)+1}}\right) \mathrm{d} x, \quad\{-1<\mathfrak{R}(s)<0\}
$$

However the iterates are associative, thus,

$$
\zeta^{\overline{n+1}}(z)=\zeta(s)=0
$$

Of course, this is only true if the backward iterations are uniquely selected from the initial argument set $0<\mathfrak{R}(z)<1$. The range of the function changes since, $\zeta^{\overline{n+2}}(z)=\zeta(0)=-\frac{1}{2}$. Thus,

$$
\left.\begin{array}{l}
0<\mathfrak{R}(s)<1, k=0, \zeta(s)=s \int_{1}^{\infty}\left(\frac{\lfloor x\rfloor-x}{x^{s+1}}\right) \mathrm{d} x+\frac{s}{s-1} \\
-1<\mathfrak{R}(Z)<0, k>0, \zeta(Z)=Z \int_{0}^{\infty}\left(\frac{\lfloor x\rfloor-x+\frac{1}{2}}{x^{Z+1}}\right) \mathrm{d} x
\end{array}\right\}
$$

$k$-iteration of the function for arguments in the range $-1<\mathfrak{R}\left(\zeta^{\vec{k}}(z)\right)<0$, give the functional relation.

$$
\zeta^{\overline{n-1}}(z) \int_{0}^{\infty}\left(\frac{\lfloor x\rfloor-x+\frac{1}{2}}{x^{\zeta^{n-1}}(z)+1}\right) \mathrm{d} x=\zeta^{\overline{n-1}}\left((z) \int_{0}^{\infty}\left(\frac{\lfloor x\rfloor-x+\frac{1}{2}}{x^{z+1}}\right) \mathrm{d} x\right)
$$


In other words, after convergence, the relation obeys the functional,

$$
f(z) g(f(z))=f(z(g(z)))
$$

Then, for $-1<\mathfrak{R}\left(\overline{\zeta^{\overline{n+2}}}(z)\right)<0$,

$$
\begin{gathered}
\zeta\left(\zeta^{\infty}(s)\right)=\zeta^{\infty}(s)=C_{\infty}, \\
C_{\infty}=C_{\infty} \int_{0}^{\infty}\left(\frac{\lfloor x\rfloor-x+\frac{1}{2}}{x^{C_{\infty}+1}}\right) \mathrm{d} x \rightarrow \int_{0}^{\infty}\left(\frac{\lfloor x\rfloor-x+\frac{1}{2}}{x^{C_{\infty}+1}}\right) \mathrm{d} x=1
\end{gathered}
$$

The relation gives the invariant integral over an infinite iteration of the roots:

$$
\int_{0}^{\infty}\left(\frac{\lfloor x\rfloor-x+\frac{1}{2}}{x^{C_{\infty}+1}}\right) \mathrm{d} x=1
$$

I now introduce the function $\varphi(x)$ defined by the following theorem due to Ramanujan. Chakravarthi Padmanabhan Ramanujan was born in India on the $9^{\text {th }}$ of January 1838 . He died on the $27^{\text {th }}$ of October 1874 . He has been referred to as the greatest mathematician ever. His work on number theory and algebraic geometry has produced some of the most outstanding revelations in mathematics and is considered to be one of the pillars of modern day research. This paper is about Ramanujan's so called Master Theorem that relates integrals of certain types of functions to a wide range of application including Power towers and the Zeta function.

The function $f(x)=\mathrm{e}^{x\left(\mathrm{e}^{-x}\right)}$ is intimately related to the Ramanujan Master Theorem and to the convergence of the Power Tower Zeta function.

LEMMA 11: [Ramanujan's Master theorem Ref. [12]].

Define $\psi_{y}(k)=\sum_{m=0}^{\infty} \frac{(m)^{k} y^{m}}{(m) !}$, then, if $f(x)=\sum_{k=0}^{\infty} \frac{\psi_{y}(k)}{k !}(-x)^{k}$ in some neighborhood of $x=0$, then,

$$
\left.\int_{0}^{\infty} f(x) x^{-\alpha-1}\right) \mathrm{d} x=\Gamma(-\alpha) \psi_{y}(\alpha)
$$

DEFINITION 9: For any real or complex numbers, $x, y$ let

$$
S(x, y)=\mathrm{e}^{x\left(\mathrm{e}^{-y}\right)}
$$

Then using the Master Theorem, the following apply:

LEMMA 12: There exists exponential power towers such that if $\psi_{y}(k)=\sum_{m=0}^{\infty} \frac{(m)^{k} y^{m}}{(m) !}$ then,

$$
S(x, y)=\sum_{k=0}^{\infty} \frac{\psi_{y}(k)}{k !}(-x)^{k}
$$

Proof: $S(x, y)=\mathrm{e}^{x\left(\mathrm{e}^{-y}\right)}$. Then,

$$
\begin{gathered}
S(x, y)=\sum_{k=0}^{\infty} \sum_{m=0}^{\infty} \frac{(x)^{m}(-m y)^{k}}{m ! k !} \\
=\sum_{k=0}^{\infty} \frac{(-x)^{k}}{k !} \sum_{m=0}^{\infty} \frac{(m)^{k}(y)^{m}}{(m) !}
\end{gathered}
$$




$$
S(x, y)=\sum_{k=0}^{\infty} \frac{\psi_{y}(k)(-x)^{k}}{k !}
$$

The following Theorem by Thron, [8], applies to such power towers.

LEMMA 13 \{Thron\}: If $a=\frac{f(x)}{\mathrm{e}^{f(x)}},|x|<1$, or if $f(x)$ is a root of unity, then the sequence (143) converges to $\mathrm{e}^{f(x)}$. For almost all $f(x)$ such that $|f(x)|=1$, the sequence diverges.

LEMMA 14: For any complex numbers, $x, y$, if $y=\ln (x)-\ln (\ln (x))$, then $S(x, y)=x$.

Proof:

$$
\begin{aligned}
& S(x, y)=\mathrm{e}^{x\left(\mathrm{e}^{-y)}\right)} \\
& =\mathrm{e}^{x \mathrm{e}^{(-\ln (x)+\ln (\ln (x)))}} \\
& =\mathrm{e}^{x\left(\mathrm{e}^{\ln \left(\frac{\ln (x)}{(x)}\right)}\right)} \\
& =\mathrm{e}^{\frac{(x)\left(\frac{\ln ((x))}{(x)}\right)}{(x)}}=\mathrm{e}^{\ln \left(((x))\left(\frac{(x)}{(x)}\right)\right.}=x
\end{aligned}
$$

LEMMA 15: For any complex numbers, $x$, if $f(x)=\ln (x+1)$, then, the self-root function is given by:

$$
S(f(x), f(x))=(x+1)^{\frac{1}{(x+1)}}=\sum_{k=0}^{\infty} \frac{(-x)^{k}}{k !} \psi_{S}(k)
$$

where,

$$
\psi_{S}(k)=(-1)^{k} \sum_{j=0}^{k}\left[\begin{array}{l}
k \\
j
\end{array}\right] \sum_{m=0}^{j} \frac{(-m)^{j-m} j !}{(j-m) !(m) !}
$$

Proof: In [12], and in [13], Jovovic calculated the self-root sum:

$$
(x+1)^{\frac{1}{(x+1)}}=\sum_{k=0}^{\infty} \frac{(x)^{k}}{k !} \sum_{j=0}^{k}\left[\begin{array}{l}
k \\
j
\end{array}\right] \sum_{m=0}^{j} \frac{(-m)^{j-m} j !}{(j-m) !(m) !}
$$

where $\left[\begin{array}{l}k \\ j\end{array}\right]$ is the Sterling number of the first kind. Thus, for the self-root,

$$
\psi_{s}(k)=(-1)^{k} \sum_{j=0}^{k}\left[\begin{array}{l}
k \\
j
\end{array}\right] \sum_{m=0}^{j} \frac{(-m)^{j-m} j !}{(j-m) !(m) !}
$$

Note that in general, one can write:

$$
S(f(x), f(x))=(x+1)^{\frac{1}{(x+1)}}=\sum_{k=0}^{\infty} \frac{n^{k}}{k !}\left(-\frac{x}{n}\right)^{k} \sum_{j=0}^{k}[k] \sum_{m=0}^{j} \frac{(-m)^{j-m} j !}{(j-m) !(m) !}
$$

In which case the function $\psi_{s}(k)$ becomes dependent on $\frac{x}{n}$.

$$
\psi_{x, n}(k)=\left(-\frac{x}{n}\right)^{k} \sum_{j=0}^{k}\left[\begin{array}{l}
k \\
j
\end{array}\right] \sum_{m=0}^{j} \frac{(-m)^{j-m} j !}{(j-m) !(m) !}
$$


LEMMA 16: If $f(x)=\ln (x+1)$, then, $S(f(x), f(x))$ is a Power Tower and, $S^{\bar{\infty}}(f(x), f(x))$ converges to $\mathrm{e}^{f(x)}$.

Proof: Use Lemma 14.

LEMMA 17: For the self-root if $f(x)=\ln (x+1)$ then,

$$
\psi_{s}(k)=\left.(-1)^{k} \frac{\mathrm{d}^{k}}{\mathrm{~d} x^{k}}(S(f(x), f(x)))\right|_{x=0}, k \in Z, k \geq 0 .
$$

\section{Proof:}

$$
S(f(x), f(x))=(x+1)^{\frac{1}{(x+1)}}
$$

Differentiating $(x+1)^{\frac{1}{(x+1)}}, k$ times, a simple calculation shows that:

$$
\left.\frac{\mathrm{d}^{k}}{\mathrm{~d} x^{k}}\left((x+1)^{\frac{1}{(x+1)}}\right)\right|_{x=0}=S^{k}(0,0)=(-1)^{k} \sum_{j=0}^{k}\left[\begin{array}{l}
k \\
j
\end{array}\right] \sum_{m=0}^{j} \frac{(-m)^{j-m} j !}{(j-m) !(m) !}
$$

where the symbol $S^{k}(0,0)$ is the value of the $k^{\text {th }}$ derivative at $x=0$. This is the same expression as $\psi_{s}(k)$ for the self-root

$$
\left.\psi_{s}(k)=(-1)^{k} \frac{\mathrm{d}^{j}}{\mathrm{~d} x^{j}}\left((x+1)^{\frac{1}{(x+1)}}\right)\right]_{x=0}=(-1)^{k} \sum_{j=0}^{k}\left[\begin{array}{l}
k \\
j
\end{array}\right] \sum_{m=0}^{j} \frac{(-m)^{j-m} j !}{(j-m) !(m) !}
$$

LEMMA 18: If $F(x)=\sum_{k=0}^{\infty} \frac{\psi_{s}(k)}{k !}(-x)^{k}$, then, $\int_{x}^{\infty} F(x) \mathrm{d} x=0$.

Proof: Using the Master theorem,

$$
\psi_{s}(k)=(-1)^{k} \sum_{j=0}^{k}\left[\begin{array}{l}
k \\
j
\end{array}\right] \sum_{m=0}^{j} \frac{(-m)^{j-m} j !}{(j-m) !(m) !}
$$

Now,

$$
F(x)=\sum_{k=0}^{\infty} \frac{\psi_{s}(k)}{k !}(-x)^{k}
$$

From the Zeta function, since $\psi_{s}(k)$ is independent of $n$,

$$
\begin{gathered}
\sum_{n=0}^{\infty} F(n)=\sum_{n=0}^{\infty} \sum_{k=0}^{\infty} \frac{\psi_{s}(k)}{k !}(-n)^{k}=\sum_{k=0}^{\infty} \frac{\psi_{s}(k)}{k !}(-1)^{k} \zeta(-k) \\
=\sum_{k=0}^{\infty} \frac{(-1)^{k} B_{k+1} \psi_{s}(k)}{(k+1) !}
\end{gathered}
$$

Now from Euler-Mac Laurin summation formula:

$$
\sum_{n=1+a}^{\infty} F(n)=\int_{a}^{b} f(x) \mathrm{d} x+\frac{1}{2}[F(a)-F(b)]+\sum_{n=0}^{\infty} \frac{B_{2 n}}{(2 n) !}\left\{F^{2 n-1}(b)-F^{2 n-1}(a)\right\}
$$

Putting $a=0, b=\infty$,

$$
\int_{0}^{\infty} F(x) \mathrm{d} x=\sum_{n=1}^{\infty} F(n)-\frac{1}{2}[F(0)]+\frac{1}{12} F^{\prime}(0)-\frac{1}{720} F^{\prime \prime \prime}(0)+\frac{1}{30240} F^{\prime \prime \prime \prime}(0)+\cdots
$$




$$
\left[\int_{0}^{\infty} F(x) \mathrm{d} x=\sum_{k=0}^{\infty} \frac{(-1)^{k} B_{k+1} \psi_{s}(k)}{\Gamma(k)}-\sum_{k=0}^{\infty} \frac{B_{k+1} F^{k}(0)}{\Gamma(k)}\right]
$$

Noting from the Bernoulli relation that only odd values of $k$ survive, the integral (171) is zero when $\psi_{s}(k)=(-1)^{k} F^{k}(0), k \geq 0$.

LEMMA 19: Define $F(x)=\lfloor x\rfloor-x+\frac{1}{2}=\sum_{m=1}^{\infty} \frac{\sin (2 \pi m x)}{m \pi}$, then, $F(x)=\sum_{k=0}^{\infty} \frac{\psi_{F}(k)}{k !}(-x)^{k}$, where

$$
\psi_{F}(k)=-\frac{\zeta(k)}{\Gamma(1-k)} .
$$

\section{Proof:}

Consider the Fourieh series expansion,

$$
\begin{gathered}
F(x)=\lfloor x\rfloor-x+\frac{1}{2}=\sum_{m=1}^{\infty} \frac{\sin (2 \pi m x)}{m \pi} \\
F(x) \leq \frac{1}{2}, x \in Z
\end{gathered}
$$

The derivatives $F^{k}(0)$ of $F(x)$ at $x=0$ are given by:

$$
F^{k}(0)=\sum_{n=1}^{\infty}-(-1)^{k} 2^{k}(\pi)^{k-1} \sin \left(\frac{\pi k}{2}\right) n^{k-1}
$$

Put:

$$
\begin{gathered}
\psi_{F}(k)=(-1)^{k} F^{k}(0)=-2^{k}(\pi)^{k-1} \sin \left(\frac{\pi k}{2}\right) \zeta(1-k), \\
\psi_{F}(k)=(-1)^{k} F^{k}(0)=-\frac{\zeta(k)}{\Gamma(1-k)},
\end{gathered}
$$

then,

$$
F(x)=\sum_{k=0}^{\infty} \frac{\psi_{F}(k)}{k !}(-x)^{k}=\sum_{m=1}^{\infty} \sum_{k=0}^{\infty} \frac{-(-1)^{k} 2^{k}(\pi)^{k-1} \sin \left(\frac{\pi k}{2}\right) m^{k-1}}{k !}(x)^{k}=\sum_{m=1}^{\infty} \frac{\sin (2 \pi m x)}{m \pi}
$$

Thus, the fractional functions $\psi_{F}(k), F(x)$ satisfy Ramanujan's Master Theorem, when $\psi_{F}(k)=(-1)^{k} F^{k}(0)$ for $-1<\mathfrak{R}(\alpha)<0$, and so

$$
F(x)=\sum_{k=0}^{\infty} \frac{(-1)^{k} \psi_{F}(k)}{k !}(x)^{k}
$$

Then,

$$
\psi_{F}(k)=-\sum_{m=0}^{\infty} \frac{m^{k-1}}{k !}
$$

*Note that putting $j-m=k-1$ in

$$
\begin{gathered}
\psi_{S}(k)=\sum_{m=1-k}^{k}\left[\begin{array}{c}
k \\
k+m-1
\end{array}\right] \sum_{m=0}^{k+m-1} \frac{(-m)^{k-1}(k-1+m) !}{(k-1) ! m !}, \\
\psi_{S}(k)=-\left[\begin{array}{l}
k \\
0
\end{array}\right](-1)^{k} \sum_{m=0}^{k+m-1} \frac{(m)^{k-1}(k-1+m) !}{(k-1) ! m !},
\end{gathered}
$$


Then, as $m \rightarrow \infty, \psi_{S}(k) \rightarrow \psi_{F}(k)$.

LEMMA 20: If $\psi_{y}(k)=\sum_{m=0}^{\infty} \frac{(m)^{k} y^{m}}{(m) !}$, then, $\int_{0}^{\infty} \psi_{y}(\alpha) \mathrm{e}^{-y} \mathrm{~d} y=\zeta(-\alpha)$.

Proof:

$$
\begin{gathered}
\mathrm{e}^{-y} \psi_{y}(\alpha)=\sum_{m=0}^{\infty} \frac{(m)^{\alpha-1} y^{m}}{(m-1) !} \mathrm{e}^{-y} \\
\mathrm{e}^{-y} \psi_{y}(\alpha)=\sum_{m=0}^{\infty} \frac{(y)^{m} \mathrm{e}^{-y}}{(m-1) !}(m)^{\alpha-1}=\sum_{m=0}^{\infty} \frac{(y)^{m} \mathrm{e}^{-y}}{(m) !}(m)^{\alpha} \\
\int_{0}^{\infty} \psi_{y}(\alpha) \mathrm{e}^{-y} \mathrm{~d} y=\sum_{m=0}^{\infty} \frac{\int_{0}^{\infty}(y)^{m} \mathrm{e}^{-y} \mathrm{~d} y}{(m) !}(m)^{\alpha}=\sum_{m=0}^{\infty} \frac{\Gamma(m+1)}{(m) !}(m)^{\alpha} \\
\int_{0}^{\infty} \psi_{y}(\alpha) \mathrm{e}^{-y} \mathrm{~d} y=\zeta(-\alpha)
\end{gathered}
$$

LEMMA 21: For complex values of $z$, if $p(z)=\sum_{k=0}^{\infty} \psi_{y}(k) \frac{(-z)^{k}}{k !}$, then

$$
\int_{0}^{\infty} \mathrm{e}^{-z} p(z) \mathrm{d} z=\sum_{m=1}^{\infty}(m)^{-m} .
$$

Proof:

$$
\begin{aligned}
& p(y)=\sum_{k=0}^{\infty} \sum_{m=0}^{\infty} \frac{(y)^{m}(-m y)^{k}}{m ! k !} \\
& \int_{0}^{\infty} p(y) \mathrm{e}^{-y} \mathrm{~d} y=\sum_{m=0}^{\infty} \sum_{k=0}^{\infty} \frac{(-1)^{k} \int_{0}^{\infty}(y)^{k+m} \mathrm{e}^{-y} \mathrm{~d} y}{k !} \frac{(m)^{k-1}}{(m-1) !} \\
&=\sum_{k=0}^{\infty} \sum_{m=0}^{\infty} \frac{(-1)^{k} \Gamma(k+m+1)}{k !} \frac{(m)^{k}}{(m) !} \\
&=\sum_{m=0}^{\infty}(m+1)^{-m-1}=\sum_{m=1}^{\infty}(m)^{-m}
\end{aligned}
$$

LEMMA 22: For complex values of $z$, if $S(f(z), f(z))=\sum_{k=0}^{\infty} \psi_{s}(k) \frac{(-z)^{k}}{k !}$ and $\psi_{s}$ is not a function of $z$, then the Ramanujan function

$$
\left[S(f(z), f(z))=\sum_{k=0}^{\infty} \frac{\psi_{S}(k)}{k !}(-z)^{k}\right]
$$

is related to the Riesz function.

Proof: Integrating (181), and since $\psi_{s}(k)$ is independent of $z$,

$$
\int_{0}^{\infty} S(f(z), f(z)) \mathrm{d} x=\lim _{x \rightarrow \infty} \sum_{k=0}^{\infty} \frac{(-1)^{k} \psi_{s}(k)}{\Gamma(k+1)} z^{k+1}
$$

Note that (182) is exactly the Riesz function for $\psi_{s}(k)=\frac{1}{\zeta(2 k+2)}$; 


$$
\int_{0}^{\infty} S(f(z), f(z)) \mathrm{d} z=\lim _{z \rightarrow \infty} \sum_{k=0}^{\infty} \frac{(-1)^{k} z^{k+1}}{\Gamma(k+1) \zeta(2 k+2)}=\lim _{x \rightarrow \infty} \sum_{k=1}^{\infty} \frac{(-1)^{k+1} z^{k}}{\Gamma(k) \zeta(2 k)}
$$

LEMMA 23: For $\left.\{-1<\mathfrak{R}(\alpha)<0\}, \zeta(\alpha)=\alpha \int_{0}^{\infty} F(x) x^{-\alpha-1}\right) \mathrm{d} x=\Gamma(1-\alpha) \psi_{F}(\alpha)$.

Proof:

If $F(x)=\sum_{k=0}^{\infty} \frac{(-1)^{k} \psi_{F}(k)}{k !}(x)^{k}$, then,

$$
\left.\int_{0}^{\infty}(F(x)) x^{-\alpha-1}\right) \mathrm{d} x=\Gamma(-\alpha) \psi_{F}(\alpha)
$$

Using the Zeta functional relation,

$$
\zeta(\alpha)=(2)^{\alpha}(\pi)^{\alpha-1} \sin \left(\frac{\pi \alpha}{2}\right) \Gamma(1-\alpha) \zeta(1-\alpha)
$$

Relation (185) becomes:

$$
\psi_{F}(\alpha)=-(2)^{\alpha}(\pi)^{\alpha-1} \sin \left(\frac{\pi \alpha}{2}\right) \zeta(1-\alpha)=-\frac{\zeta(\alpha)}{\Gamma(1-\alpha)}
$$

For $-1<\mathfrak{R}(\alpha)<0$, the function $\psi_{F}(\alpha)$ is related to the Zeta function in an intimate way and so

$$
\zeta(\alpha)=\alpha \int_{0}^{\infty} F(x) x^{-\alpha-1} \mathrm{~d} x=-\psi_{F}(\alpha) \Gamma(1-\alpha)
$$

Corollary: If $F(x)=\sum_{k=0}^{\infty} \frac{(-1)^{k} \psi_{F}(\alpha)}{k !}(x)^{k}$, then

$$
\zeta(\alpha)=\alpha \int_{0}^{\infty} F(x) x^{-\alpha-1} \mathrm{~d} x=-\psi_{F}(\alpha) \Gamma(1-\alpha)
$$

Proof:

$$
\zeta(\alpha)=\alpha \int_{0}^{\infty} \sum_{k=0}^{\infty} \frac{(-1)^{k} \psi_{F}(\alpha)}{k !} x^{k-\alpha-1} \mathrm{~d} x
$$

Thus $\psi_{F}(k)=\psi_{F}(\alpha)=$ constant, then,

$$
\zeta(\alpha)=\alpha \int_{0}^{\infty} \sum_{k=0}^{\infty} \frac{(-1)^{k} \psi_{F}(\alpha)}{k !} x^{k-} \mathrm{d} x=\alpha \int_{0}^{\infty} \mathrm{e}^{-x} x^{-\alpha-1} \mathrm{~d} x \psi_{F}(\alpha)=-\psi_{F}(\alpha) \Gamma(1-\alpha)
$$

It is worth noting that relation (190) is one form of the Weyl fractional derivative. See Ref. [12]. The Riemann-Louiville fractional integral gives the same result.

$$
\begin{gathered}
\psi_{F}(\alpha)=-\frac{\zeta(\alpha)}{\Gamma(1-\alpha)} \\
\psi_{F}(\alpha)=-(2)^{\alpha}(\pi)^{\alpha-1} \sin \left(\frac{\pi \alpha}{2}\right) \zeta(1-\alpha)=-\frac{\zeta(\alpha)}{\Gamma(1-\alpha)}
\end{gathered}
$$

\section{Relationship of the Function $\psi_{F}(\alpha)$ to the Density of Squarefree and Squarefull (Non-Squarefree) Numbers}

DEFINITION 10: A positive integer $m$ is squarefree if it is either a product of different primes or 1 otherwise it 


\section{is squarefull.}

Let $\chi(1), \vartheta(1)$ be the densities of number of squarefree numbers and squarefull numbers in an infinite set of integers.

Note: RIEMANN'S HYPTOTHESIS: Fix $\epsilon>0$. Then we can find $N$ such that for all $n>N$ the number of square free numbers in $[1, n]$ does not differ from the number of non-square numbers in $[1, n]$ by more than $n^{\frac{1}{2}+\epsilon}$.

LEMMA 24: The density of squarefree numbers is $\chi(1)=\frac{\pi^{2}}{6}$, and the density of squarefull numbers is $\vartheta(1)=1-\chi(1)$.

\section{Proof:}

Taken over square-free numbers $m$, and squarefull numbers $n$, then:

$$
\psi_{F}(\alpha)=(-1)^{\alpha} F^{\alpha}(0)=-\sum_{m=\text { squarefree }}^{\infty} 2^{\alpha-1}(\pi)^{\alpha} \sin \left(\frac{\pi \alpha}{2}\right)(m)^{\alpha-1}-\sum_{n=\text { squarefull }}^{\infty} 2^{\alpha-1}(\pi)^{\alpha} \sin \left(\frac{\pi \alpha}{2}\right)(n)^{\alpha-1}
$$

Since

$$
\begin{gathered}
-2^{\alpha-1}(\pi)^{\alpha} \sin \left(\frac{\pi \alpha}{2}\right) \Gamma(1-\alpha) \sum_{m=\text { squarefree }}^{\infty} n^{-\alpha}=-\frac{\zeta(\alpha)}{\zeta(2 \alpha)} \\
\psi_{F}(\alpha) \Gamma(1-\alpha)=-\zeta(\alpha)=-\frac{\zeta(\alpha)}{\zeta(2 \alpha)}-\left(\Gamma(1-\alpha) \sum_{n=\text { squarefull }}^{\infty} 2^{\alpha-1}(\pi)^{\alpha} \sin \left(\frac{\pi \alpha}{2}\right)(n)^{\alpha-1}\right) \\
\frac{\zeta(\alpha)}{\Gamma(1-\alpha)}\left(1-\frac{1}{\zeta(2 \alpha)}\right)=\sum_{n=\text { squarefull }}^{\infty} 2^{\alpha-1}(\pi)^{\alpha} \sin \left(\frac{\pi \alpha}{2}\right)(n)^{\alpha-1}
\end{gathered}
$$

Thus,

$$
\psi_{F}(k)\left(\frac{\zeta(2 k)-1}{\zeta(2 k)}\right)=\sum_{n=\text { squarefull }}^{\infty} 2^{\alpha-1}(\pi)^{\alpha} \sin \left(\frac{\pi \alpha}{2}\right)(n)^{\alpha-1}
$$

Let

$$
\begin{aligned}
& \vartheta(\alpha)=-\sum_{n=\text { squarefull }}^{\infty} 2^{\alpha-1}(\pi)^{\alpha} \sin \left(\frac{\pi \alpha}{2}\right)(n)^{\alpha-1} \\
& \chi(\alpha)=-\sum_{m=\text { squarefree }}^{\infty} 2^{\alpha-1}(\pi)^{\alpha} \sin \left(\frac{\pi \alpha}{2}\right)(m)^{\alpha-1}
\end{aligned}
$$

Then,

$$
\psi_{F}(\alpha)\left(\frac{\zeta(2 \alpha)-1}{\zeta(2 \alpha)}\right)=\vartheta(\alpha)
$$

The number of both the squarefree and the square-full numbers is given by $\psi_{c}(1)$ for $k=1$, since this is the one by one count of each infinite set. Thus, the density of the squarefree numbers and the squarefull numbers is given by:

$$
\psi_{F}(1)=\chi(1)+\vartheta(1)=2^{1}(\pi)^{0} \sin \left(\frac{\pi}{2}\right) \zeta(1-1)=1
$$

thus,

$$
\left(\frac{\zeta(2)-1}{\zeta(2)}\right)=\vartheta(1)
$$




$$
\vartheta(1)=1-\frac{6}{\pi^{2}}, \chi(1)=\frac{6}{\pi^{2}}
$$

It follows that the density of square-free numbers is $\frac{6}{\pi^{2}}$, and the density of squarefull numbers is $\vartheta(1)=1-\frac{6}{\pi^{2}}$.

LEMMA 25: Let $g_{n}(x)=\mathrm{e}^{-n^{2} \pi x}$, then, $\int_{0}^{\infty} g_{n}(x) \mathrm{d} x=0$ and $g_{n}(x)=\sum_{k=0}^{\infty} \frac{\psi_{g}(k)}{k !}(-x)^{k}$.

Proof:

Taking the derivatives, $g_{n}^{k}(0)=(-1)^{k} \pi^{k} n^{2 k}=(-1)^{k} \psi_{g}(k)$;

$$
g_{n}(x)=\sum_{k=0}^{\infty} \frac{\pi^{k} n^{2 k}}{k !}(-x)^{k}=\mathrm{e}^{-n^{2} \pi x}=g_{n}(x)
$$

One sees that the paired functions $g_{n}(x), \psi_{g}(k)$ satisfy the Master Theorem. Thus, from LEMMA 25 $\int_{0}^{\infty} g_{n}(x) \mathrm{d} x=0$.

Further, to see that this is true, define:

$$
\begin{gathered}
S(x)=\sum_{n=1}^{\infty} \mathrm{e}^{-n^{2} \pi x}=\sum_{n=1}^{\infty} g_{n}(x) \\
\int_{0}^{\infty} g_{n}(x) x^{-\alpha-1} \mathrm{~d} x=\int_{0}^{\infty} \mathrm{e}^{-n^{2} \pi x} x^{-\alpha-1} \mathrm{~d} x=\pi^{\alpha} n^{2 \alpha} \Gamma(-\alpha)=\psi_{g}(\alpha) \Gamma(-\alpha) \\
\pi^{-\frac{s}{2}} \zeta(s) \Gamma\left(\frac{s}{2}\right)=\int_{0}^{\infty} S(x) x^{\frac{s}{2}-1} \mathrm{~d} x
\end{gathered}
$$

Put $s=-2 \alpha$,

$$
\begin{gathered}
\pi^{\alpha} \zeta(-2 \alpha) \Gamma(-\alpha)=\int_{0}^{\infty} \sum_{n=1}^{\infty} g_{n}(x) x^{\alpha-1} \mathrm{~d} x=\sum_{n=1}^{\infty} \pi^{\alpha} n^{2 \alpha} \Gamma(-\alpha) \\
\pi^{\alpha} \zeta(-2 \alpha) \Gamma(-\alpha)=\pi^{\alpha} \zeta(-2 \alpha) \Gamma(-\alpha)
\end{gathered}
$$

LEMMA 26: If a function, $f(x)$, has derivatives of all orders throughout a neighborhood of a point $\xi$, then $\psi_{c}(k)=(-1)^{k} f^{k}(0)$ and Ramanujan's Master Theorem is simply the Taylor series.

Proof: The Taylor series of any function $f(x)$ that has derivatives of all orders throughout a neighborhood of a point $\xi$, may be written as:

$$
f(x)=\sum_{k=0}^{\infty} \frac{(x-\xi)^{k}}{k !} f^{k}(\xi)
$$

Thus as $\xi \rightarrow 0$,

$$
f(x)=\sum_{k=0}^{\infty} \frac{\left(x^{k}\right)}{k !} f^{k}(0)
$$

\section{Relation of Power Towers to Transcendental Numbers}

As can be seen from Figure 6, the function $x^{\frac{1}{x}}$ only has quadratically equal values (red $x$ 's) at $x=2,4$, and also at $x=1, \infty$. There are no other paired quadratically equal values of $x$ that have the same values for $x^{\frac{1}{x}}$ Hence the following LEMMA due to Mladen [14]. 


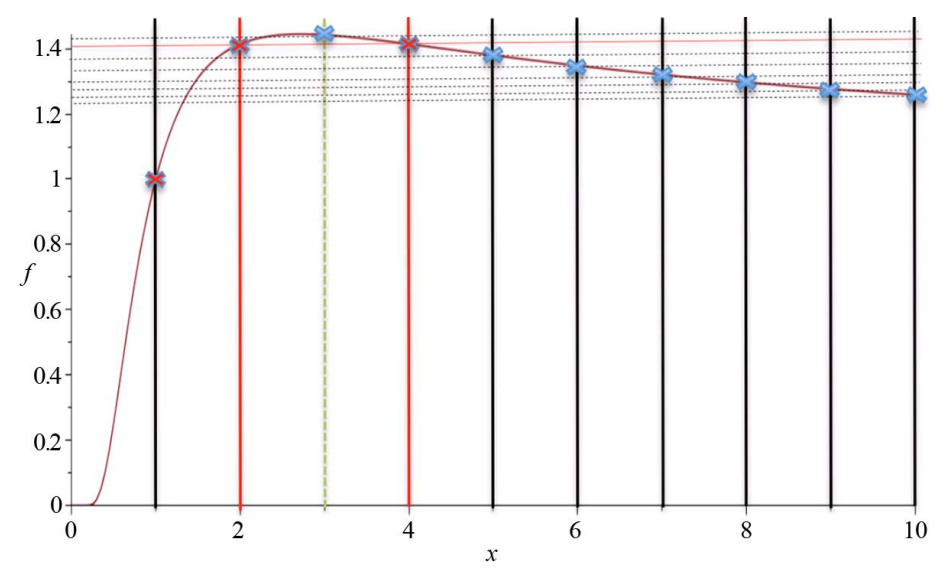

Figure 6. Shows a plot of the function $S(f(x), f(x))=x^{\frac{1}{x}}$.

\section{LEMMA 27:}

(Mladen, [14]) For every real number $x \geq 1$, if $f(x)=\ln (x+1)$ then, $S(f(x), f(x))=\sqrt[x+1]{x+1}$, and

a) $S:(0, \infty) \rightarrow(1, \sqrt[e]{e})$

b) $S(1,1)=1 ; S(e, e)=\sqrt[e]{e} ; \lim _{x \rightarrow+\infty} S(f(x), f(x))=1$.

c) $S(f(x), f(x))$ is a continuous function strictly increasing on the interval $x=(0, e-1)$ and strictly decreasing on the interval $(e-1, \infty)$ and $S(f(x), f(x))$ has an absolute maximum at $x=e-1$, i.e. for $x \in(0, e-1)$ :

$$
\sqrt[e]{e}=S(f(e), f(e))>S(f(x), f(x))>S(f(1), f(1))=1,
$$

and for $x \in(e, \infty)$,

$$
\lim _{x \rightarrow \infty} S(f(x), f(x))=1 .
$$

LEMMA 28: Let $f(x)=\ln (x), S(f(x), f(x)) \in(1,+\infty)$ be a real number, then:

a) For $S(f(x), f(x)) \in(1, \sqrt[e]{e})$ the equation

$$
S(f(x), f(x))=\sqrt[x]{x}
$$

has exactly two solutions, $x_{1} \in(1, e)$ and $x_{2} \in(e,+\infty)$.

b) For $S(f(x), f(x))=\sqrt[e]{e}$, (207) has the unique solution $x=e$.

c) For $S(f(x), f(x))>\sqrt[e]{e}$, (207) has no solutions.

LEMMA 29: Let $x>1$ be an algebraic number such that $f(x)=\ln (x)$, then,

$$
S(f(x), f(x))=\sqrt[x]{x},
$$

a) if $x \in(1, e)$, then, the infinite Power Tower $S^{\bar{\infty}}(f(x), f(x))=\sqrt[x]{x} \sqrt[\sqrt[x]{x}]{\sqrt[x]{x}}=\alpha_{x}$ is a rational number, and

$$
S^{\bar{\infty}}(f(x), f(x))=x
$$

b) if $x=\left(\frac{m+1}{m}\right)^{m+1}=S\left(\ln \left(\frac{m+1}{m}\right), \ln \left(\frac{1}{m+1}\right)\right)$, for some integer $m \geq 1$, then $x \in(e, \infty)$,

$$
S(f(x), f(x))=\left(\frac{1+m}{m}\right)^{m} \sqrt{\left(\frac{1+m}{m}\right)^{m}}=S\left(f\left(\left(\frac{1+m}{m}\right)^{m}\right), f\left(\left(\frac{1+m}{m}\right)^{m}\right)\right)
$$


and the infinite Power Tower $S^{\bar{\infty}}(f(x), f(x))=\sqrt[x]{x} \sqrt[x]{x}^{\sqrt[x]{x}}=\alpha_{x}$ is a rational number given by:

$$
S^{\bar{\infty}}(f(x), f(x))=\left(\frac{1+m}{m}\right)^{m}
$$

c) If $n \in(e, \infty)$ and $n$ is an integer, then it is not part of the sequence

$$
n=\left\{S\left(\ln \left(\frac{1+m}{m}\right), \ln (1+m)\right)\right\}_{m=1}^{\infty}=\left\{\left(\frac{m+1}{m}\right)^{m+1}\right\}_{m=1}^{\infty},
$$

then the infinite Power tower $S^{\bar{\infty}}(f(n), f(n))=\sqrt[n]{n} \sqrt[n n^{n}]{\sqrt[n]{n}}=\beta_{n}$ is a transcendental number.

Proof of Theorem 1: For $\mathfrak{R}(s)>0$, the Zeta function vanishes when $\mathfrak{R}(s)=\frac{1}{2}$.

Let $S^{\bar{\infty}}(f(n), f(n))=\sqrt[n]{n^{\sqrt[n]{n}}}=\beta_{n}$.

Starting with the Zeta function for $\mathfrak{R}(s)>0$ :

$$
\begin{gathered}
\zeta(s)=\frac{1}{1-2^{1-s}} \sum_{n=1}^{\infty}(-1)^{n+1}(n)^{-s} \\
\zeta(s)=\frac{1}{1-2^{1-s}} \sum_{n=1}^{\infty}(-1)^{n+1}\left(n^{\frac{1}{n}}\right)^{-n s} \\
\zeta(s)=\frac{1}{\left(1+2^{\sqrt{1-s}}\right)\left(1-2^{\sqrt{1-s}}\right)} \sum_{n=1}^{\infty}(-1)^{n+1}\left(S^{\bar{\infty}}(f(n), f(n))\right)^{-n s}, k<\infty
\end{gathered}
$$

Let $\eta_{n}$ be algebraic numbers such that $\eta_{n}=-\ln \left(\beta_{n}\right) \rightarrow \mathrm{e}^{-\eta_{n}}=\beta_{n}$, then according to Lindemann-Weierstrass theorem, $\beta_{n}$ is transcendental.

Taking $k \rightarrow \infty$,

$$
S^{\tilde{\infty}}(f(n), f(n))=\frac{W\left(-\ln \left(\beta_{n}\right)\right)}{-\ln \left(\beta_{n}\right)}
$$

The sequence of integers $x=n=1,2,4$ in the Zeta-function are part of the rational sequence $\alpha_{n}$.

The sequence of integers $x=n=3,5,6,7, \cdots, \infty$ in the Zeta-function are a part of the rational sequence $\beta_{n}$, hence using LEMMA 29 the Zeta function (207) can be written as a function of transcendental numbers, $\beta$, in the form:

$$
\begin{gathered}
\zeta(s)=\frac{1}{1-2^{1-s}}\left(1-\left(g^{\bar{\infty}}\left(a_{2}\right)\right)^{-s}-\left(g^{\bar{\infty}}\left(a_{4}\right)\right)^{-s}+\sum_{n \neq 1,2,4}^{\infty}(-1)^{n+1}\left(\frac{W\left(-\ln \left(\beta_{n}\right)\right)}{-\ln \left(\beta_{n}\right)}\right)^{-s}\right) \\
\zeta(s)=\frac{1}{1-2^{1-s}}\left(1-(2)^{-s}-(2)^{-s}+\sum_{n \neq 1,2,4}^{\infty}(-1)^{k+1}\left(\frac{W\left(-\ln \left(\beta_{n}\right)\right)}{-\ln \left(\beta_{n}\right)}\right)^{-s}\right) \\
\zeta(s)=1+\frac{1}{1-2^{1-s}}\left(\sum_{n \neq 1,2,4}^{\infty}(-1)^{n+1}\left(\frac{W\left(-\ln \left(\beta_{n}\right)\right)}{-\ln \left(\beta_{n}\right)}\right)^{-s}\right)
\end{gathered}
$$

Factoring; 


$$
\begin{gathered}
\zeta(s)=1+\frac{1}{\left(1+2^{\sqrt{1-s}}\right)\left(1-2^{\sqrt{1-s}}\right)} \sum_{n \neq 1,2,4}^{\infty}(-1)^{n+1}\left(\frac{-\ln \left(\beta_{n}\right)}{W\left(-\ln \left(\beta_{n}\right)\right)}\right)^{s} \\
W\left(-\ln \left(\beta_{n}\right)\right)=-\ln \left(\beta_{n}\right) \mathrm{e}^{-W\left(-\ln \left(\beta_{n}\right)\right)}=-\ln \left(\beta_{n}\right) \mathrm{e}^{-W\left(\eta_{n}\right)} \\
\zeta(s)=1+\frac{1}{\left(1+2^{\sqrt{1-s}}\right)\left(1-2^{\sqrt{1-s}}\right)} \sum_{n \neq 1,2,4}^{\infty}(-1)^{n+1}\left(\frac{-\ln \left(\beta_{n}\right)}{\left.-\ln \left(\beta_{n}\right) \mathrm{e}^{-W\left(\eta_{n}\right)}\right)^{s}}\right. \\
\zeta(s)=1+\frac{1}{\left(1-2^{\sqrt{1-s}}\right)\left(1+2^{\sqrt{1-s}}\right)} \sum_{n \neq 1,2,4}^{\infty}(-1)^{n+1} \mathrm{e}^{-s W\left(\eta_{n}\right)}
\end{gathered}
$$

By the Lindemann-Weierstrass theorem, $n W\left(\eta_{n}\right)$ is transcendental since $\eta_{n}$ is algebraic, and so if $s$ is rational, then $\mathrm{e}^{-s W\left(\eta_{n}\right)}$ is algebraic. If $s$ is rational, when the Zeta function vanishes the sum

$$
A=\sum_{n \neq 1,2,4}^{\infty}(-1)^{n+1} \mathrm{e}^{-s W\left(\eta_{n}\right)}
$$

is algebraic and the product of a transcendental number $\left(1+2^{\sqrt{1-s}}\right)$ and a non-zero algebraic number is transcendental.

Thus we are left with the relationship:

$$
\left(2^{\sqrt{1-s}}+1\right)=\frac{A}{\left(1-2^{\sqrt{1-s}}\right)}
$$

where, $A$ is an algebraic number. See Ref. [15].

The only value for which the left hand side and the right hand sides are transcendental is when $\mathfrak{R}(s)=\frac{1}{2}$. Q.E.D.

\section{Discussion}

The Power Tower of the pure complex form with $\theta$ a rational number:

$$
S(2 n i \theta, 2 n i \theta)=\mathrm{e}^{2 n i \theta \mathrm{e}^{-2 n i \theta}}
$$

not only follow Ramanujan's Master Theorem, it also converge to exponential functions of the form: $\phi^{\vec{n}}(z)=\mathrm{e}^{2 n i \theta}$. Thus the Zeta function takes the form:

$$
\zeta^{\vec{n}}(z)=s=\in \sum_{n=1}^{\infty} \phi^{\vec{n}}(z)=\frac{\mathrm{e}^{2 i \theta}}{\mathrm{e}^{2 i \theta}-1}
$$

It is clear that the function converges only when

$$
s \in \frac{\mathrm{e}^{2 i \theta}}{\mathrm{e}^{2 i \theta}-1} \rightarrow \mathrm{e}^{2 i \theta} \in \frac{s}{s-1}
$$

This just shows the self-similarity of the convergence.

Now the only arguments $s$ that satisfy the path to convergence are:

$$
\mathrm{e}^{2 i \theta}=\frac{\frac{1}{2}+i \frac{1}{2} \tan (\theta)}{\frac{1}{2}-i \frac{1}{2} \tan (\theta)}=\frac{s}{1-s}, \mathrm{e}^{-2 i \theta}=\frac{\frac{1}{2}-i \frac{1}{2} \tan (\theta)}{\frac{1}{2}+i \frac{1}{2} \tan (\theta)}=\frac{1-s}{s}
$$


See Ref. [17], Ref. [6]. Thus, the solutions to the convergence are on the half-line.

There are an infinite number of possible roots that can independently satisfy (218). For example, if a root is obtained from the backward iteration, $s_{m}=\zeta^{\bar{m}}\left(c_{m}\right)$, then, starting at any iteration point $n<m$,

$$
\zeta^{\bar{m}}\left(c_{m}\right)=\zeta^{\overline{m-1}}\left(c_{m-1}\right)=\cdots=\zeta^{\overline{1}}\left(c_{1}\right)=0 .
$$

Thus any complex root $s$ can be obtained by iterations that go backwards from the real negative line provided the arguments are chosen to be of the rational form:

$$
\zeta^{-1}(0) \in\left\{s=\frac{\mathrm{e}^{2 i \theta}}{\mathrm{e}^{2 i \theta}-1}\right\} \in\left\{\frac{1}{2}+i \tau\right\}
$$

The solutions to inverse Zeta values is referred to as "a-points". These points have been extensively studied by other authors, see [17]. The conditions of convergence of complex power towers of the form:

$$
f(x)=\mathrm{e}^{x \mathrm{e}^{-x}}
$$

also require that

$$
\mid\left(\ln \left(\frac{\frac{1}{2}+i \frac{1}{2} \tan (\theta)}{\frac{1}{2}-i \frac{1}{2} \tan (\theta)}=\frac{s}{s-1}\right) \mid<1 .\right.
$$

The roots of the zeta function obey this condition as can be seen from Table 2:

Remark 2: The arguments that lead to roots have singular solutions to the inverse zeta functions of roots that generate them in the backward direction. This points to the fact that a root can only be arrived at when there are no cycles prior to the root. Thus, any argument $z$ that leads to a root can be backward generated for as singular values of the inverse zeta function that it generates. Not all values of $z$ can generate a root. Those that generate a root must conform to the power tower structure. The only reciprocal relations that relate the symmetry is $s \rightarrow 1-s \in \mathbb{C}$ to reciprocal functions are conjugate complex functions of the exponential forms on the half-line:

$$
\mathrm{e}^{2 i \theta}=\frac{\frac{1}{2}+i \frac{1}{2} \tan (\theta)}{\frac{1}{2}-i \frac{1}{2} \tan (\theta)}=\frac{s}{1-s}, \mathrm{e}^{-2 i \theta}=\frac{\frac{1}{2}-i \frac{1}{2} \tan (\theta)}{\frac{1}{2}+i \frac{1}{2} \tan (\theta)}=\frac{1-s}{s}
$$

From this, one could surmise that the reflection formula and the inverse zeta power towers prescribe conjugate power towers that are also reflection symmetries about unity for the Zeta function. Write the Zeta Power Tower as follows:

Table 2. The convergence condition for several non-trivial roots of the Zeta
Power Tower.
\begin{tabular}{cc}
$S$ & $\left|\left(\ln \left(\frac{s}{1-s}\right)\right)\right|$ \\
\hline$\frac{1}{2}+i 14.133725$ & 0.07071826295 \\
$\frac{1}{2}+21.022039 i$ & 0.04756015651 \\
$\frac{1}{2}+25.010857 i$ & 0.03997731031 \\
$\frac{1}{2}+98.83119 i$ & 0.01011817652 \\
$\frac{1}{2}+\infty i$ & 0.00
\end{tabular}




$$
x^{\zeta^{\vec{n}}(z)}=x^{\sum_{a_{n}=1}^{\infty} \sum_{b_{n}=1}^{\infty}\left(\left(\varnothing_{n}\right)\right)}=\prod_{b_{n}=1 a_{n}=1}^{\infty} \prod^{\infty} x^{\left(\varnothing_{n}\right)}=g(z) \in \mathbb{C}
$$

It is clear that $g(z)=1$, and the values of $x$ are just the products of the $\left(\varnothing_{n}\right)^{\text {th }}$ roots of unity when the The degree of $\mathrm{e}^{\frac{2 \pi i}{k}}$ goes to infinity with $k$. If $g(z)$ had an infinity of roots of unity, it would have elements of arbitrarily high degree, and thus would not be of finite degree over the rationals, and thus would not, in fact, be an algebraic number field. Thus, equating the powers of (223), if $\varphi_{n}$ is divisible by all the factors of $\phi_{n}$ then, the power towers can be written as

$$
\phi_{n}^{\phi_{n}^{\phi_{n}}}=\mathrm{e}^{2 i n \theta}
$$

These Power Towers are entire functions. An Entire Functions has special properties that relate to exponential functions. If $f(z)$ is analytic for the entire complex plane, then it is an entire function. An entire function can be represented by in the linear form:

$$
\sum_{k=0}^{\infty} a_{n} z^{k},|z|<\infty
$$

Anne Beurling demonstrated in all cases the translates of an integrable function defined in the entire interval $[0, \infty]$, are represented by at least one exponential form $\mathrm{e}^{-i a x}, x \geq 0, i a<0$ and thus will always contain a continuous banded group character if the function does not vanish identically.

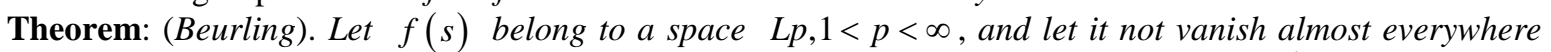
on any interval $0<s \leq a$. Then Lp contains at least one exponential function of the form $\mathrm{e}^{\lambda}$.

The function $\zeta(s)$ can also be represented by exponential terms and, rational functions of its roots can also be represented by the exponential forms. Since the function is analytic everywhere except for a simple pole with residue 1 at $s=1$, the function

$$
f(s)=\zeta(s)+\frac{s}{1-s}
$$

is an entire function, and so one suspects that when the function vanishes, there exists an exponential representation of its roots $\rho=\sigma+i \tau$ in the rational form:

$$
f(s)=\frac{\sigma+i \tau}{1-\sigma-i \tau}=\mathrm{e}^{2 i \theta}
$$

Any form of $\zeta(s)$ that expresses its roots as an entire function can be used to relate to exponential forms. There are many forms of the function that can be represented as rational functions of the roots when it vanishes. The exponential function is represented by the quotient of conjugate rational complex functions on the half-line:

$$
\mathrm{e}^{2 i \theta}=\frac{\frac{1}{2}+\frac{i}{2} \tan \theta}{\frac{1}{2}-\frac{i}{2} \tan \theta}
$$

In all these instances one finds that if the roots are on the 1/2-line, and $s=\frac{1}{2}+i \tau$, then the rational-functions $\frac{1}{2}+i \tau$

$\frac{2}{\frac{1}{2}-i \tau}$, of the roots will give three possible but distinct cases for the exponential arguments $\theta$, satisfying $\frac{1}{2}-i \tau$

$s=\frac{1}{2}+\frac{i}{2} \tan \theta$, where, $2 \tau=\arctan (\theta)$ :

$$
\frac{\frac{1}{2}+i \tau}{\frac{1}{2}-i \tau}= \begin{cases}\mathrm{e}^{2 i \alpha} & \text { Real arguments } \\ \mathrm{e}^{-2 \beta} & \text { Pure complex arguments } \\ \mathrm{e}^{2 i(\alpha+i \beta)} & \text { Complex arguments }\end{cases}
$$


with

$$
\theta=\frac{1}{2 i} \log \left(\frac{\frac{1}{2}+\frac{i}{2} \tan \theta}{\frac{1}{2}-\frac{i}{2} \tan \theta}\right)=\tan ^{-1}(\tan \theta)
$$

For the real arguments one finds that if the roots are on the 1/2-line then they must obey a certain symmetry that satisfies:

$$
\begin{gathered}
\frac{\frac{1}{2}+i \tau}{\frac{1}{2}-i \tau}=\frac{\frac{1}{2}+\frac{i}{2} \tan \alpha}{\frac{1}{2}-\frac{i}{2} \tan \alpha}=\mathrm{e}^{2 i \alpha} \\
\alpha=\frac{1}{2 i} \log \frac{\frac{1}{2}+\frac{i}{2} \tan \alpha}{\frac{1}{2}-\frac{i}{2} \tan \alpha}=\tan ^{-1} \tan \alpha, \alpha \leq \frac{\pi}{2}
\end{gathered}
$$

If the complex roots obey the exponential relation (232) for the arguments, $-\pi / 2<\alpha<\pi / 2$, then only the fractional parts of the arguments contribute to the solution of (232) and thus all the complex roots of the function will be on the $1 / 2$-line.

For the pure complex arguments, the roots will not satisfy the symmetry (223) since

$$
\beta=-\frac{1}{2 i} \log \frac{\frac{1}{2}+\frac{i}{2} \tan i \beta}{\frac{1}{2}-\frac{i}{2} \tan i \beta} \neq \tan ^{-1} \tan i \beta,
$$

Exponential-functions with pure complex argument will not be found since the right-hand side is real while the left-hand side is pure complex. For solutions with arguments that are complex, if the roots are on the half line, they must satisfy the symmetry:

$$
\alpha+i \beta=-\frac{1}{2 i} \log \frac{\frac{1}{2}+\frac{i}{2} \tan (\alpha+i \beta)}{\frac{1}{2}-\frac{i}{2} \tan (\alpha+i \beta)}=\tan ^{-1} \tan (\alpha+i \beta),
$$

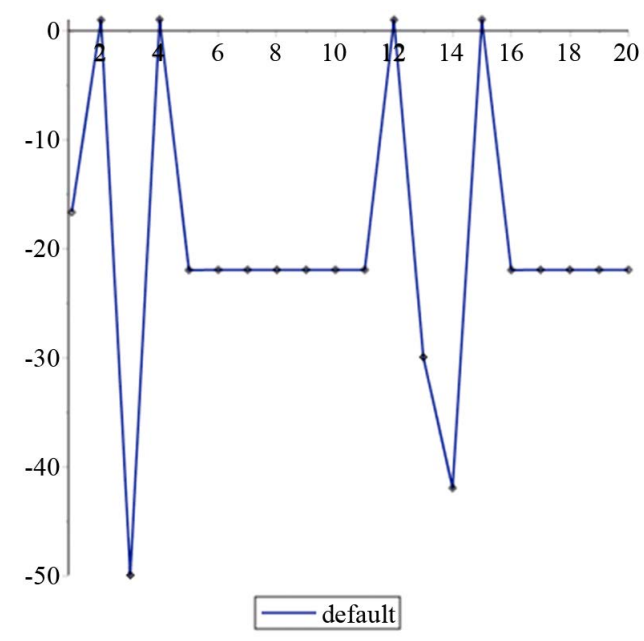

Figure 7. Values of iterations of the root $s=-2$. 
$\mathbf{0 . 7 8 0 7 5 0 8 8 2 5 9 3 1 3 7 4 9 8 6 8 6 4 7 0 1 1 7 6 4 3 1 5 8 2 3 9 7 7 0 9 3 5 3 6 9 2 0 9 4 0 0 5 5 5 6 2 9 3 6 6}$ $-42.0000000000000000037103829470559031780723007455282666145481$ 0.976512314920661294825052107822641842606733219808156231802742 $\mathbf{- 2 2 . 0 0 0 6 3 0 3 0 2 5 0 8 1 2 9 0 0 1 1 2 5 3 3 0 9 3 9 1 7 2 7 8 7 0 2 6 0 3 4 4 7 7 0 7 9 5 7 1 8 5 8 4 7 6 3 8 2 1}$ $\mathbf{- 2 1 . 9 8 5 5 2 1 8 7 1 3 5 3 7 5 9 7 5 6 8 1 6 2 5 6 0 1 0 3 6 1 2 2 8 1 7 8 7 4 2 4 6 4 6 9 2 4 5 8 6 3 4 8 2 4 0 7 4 5}$ -21.9855320025224369786834301184966320824407647441307410460570 -21.9855319957289306453932082163062285993794575904067810484136 $-21.9855319957334860654222004064588181365314389615372951431951$ -21.9855319957334830107622615867033406196779269517855306857148 -21.9855319957334830128105792571805159516160085178019531668656 $\mathbf{0 . 9 5 5 6 7 2 7 9 6 9 6 4 4 6 2 5 0 0 5 4 4 0 9 6 6 9 6 0 2 5 7 8 9 5 8 5 0 6 9 9 8 3 7 0 1 8 5 0 8 0 3 7 1 1 3 5 4 2 7 6}$ $-\mathbf{3 0 . 0 0 0 0 0 0 0 0 6 3 5 4 4 0 9 3 8 5 5 1 5 4 2 6 8 0 9 7 5 1 6 9 5 2 8 3 5 9 6 3 6 8 5 9 9 9 5 7 4 9 8 4 9 3 5 1 0 5}$ $-41.9999999999999998574302112018735692243847004551577693931243$ 0.976512314920661294744350276007417719133070635625358571510304 $-\mathbf{2 2 . 0 0 0 6 3 0 3 0 2 5 0 8 1 2 9 0 0 1 1 2 5 2 7 8 8 9 0 9 6 6 6 3 6 1 4 8 1 4 0 1 2 4 3 1 7 9 9 0 4 5 6 3 7 6 3 8 5 1}$ -21.9855218713537597568162560452633382547470627988244334229850 $-21.9855320025224369786834301184732282964315883425738984879649$ $-21.9855319957289306453932082163062442929065274986429264147673$ $-21.9855319957334860654222004064588181260080650170631918695612$ $-21.9855319957334830107622615867033406196849834534044420845689$

Figure 8 shows the iterations of the zeta function leading to the root $s=-4$ at the top of the list. Note that the iterations seem to originate from a near periodic cycle near negative roots at the bottom of the list to the root at the top.

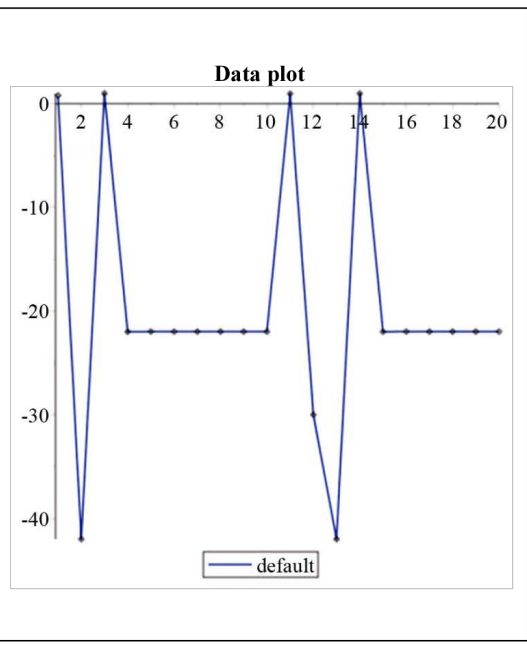

Figure 8. Values of iterations of the root $s=-4$.

-28.0000008791766792468965482318967466213065769059740983040258 $-36.0000000000081682336552883345254912489141102646962848033847$ $-60.000000000000000000000000000000067289879181098286976320048$ $\mathbf{0 . 9 8 3 4 9 1 8 1 5 2 8 7 6 1 6 7 6 9 1 9 1 4 4 3 4 2 5 3 8 7 9 5 1 2 0 3 2 7 4 5 6 2 0 4 5 1 6 3 5 3 2 3 7 3 6 4 8 5 8 6}$ $\mathbf{- 1 9 . 9 9 2 4 9 8 7 1 7 3 8 8 7 7 0 1 5 1 6 2 1 8 8 5 0 7 4 7 4 7 7 3 1 6 3 6 7 8 8 2 7 5 5 3 5 2 7 1 8 4 1 5 9 5 0 4 2 7}$ 0.951376441531459095066293058511419459604252503079156168253367 $-22.0006140909287691612244910756673865816725326068686078397431$ -21.9855218822248033746513834988680799553286948509822583417330 $-21.9855320025151473455358464706917360346432901248597843070622$ 0.955672796977789717085781341003548479288442548910148442431321 $-30.0000000063544093856040414373670011360283638740769480965198$ -41.9999999999999998574302112018735692239635748304623435194340 0.976512314920661294744350276007417719132838303321316340624930 -22.0006303025081290011252788909666361481401241681490044006039 $-21.9855218713537597568162560452633382547470627989249130213721$ $-21.9855320025224369786834301184732282964315883425738311108633$ -21.9855319957289306453932082163062442929065274986429264599474 0.955672796964453554305623331712941019386860441333575197452748 $-\mathbf{3 0 . 0 0 0 0 0 0 0 0 6 3 5 4 4 0 9 3 8 5 5 1 5 3 6 7 3 2 4 8 9 3 1 4 3 2 1 4 9 2 5 6 2 1 3 2 3 3 6 8 1 0 7 0 9 0 6 0 2 9}$ 0.967293357486602932598422796742587273359831451581003838595915

Figure 9 shows the iterations of the zeta function leading to the root $s=-6$ at the top of the list. Note that the iterations seem to originate from a non-periodic value near a negative root $s=-30$ at the bottom of the list to the root at the top.

Figure 9. Values of iterations of the root $s=-6$.

\begin{abstract}
$\mathbf{0 . 8 8 3 2 9 5 5 3 2 9 7 3 1 5 5 7 5 2 3 5 7 7 2 9 1 6 9 1 5 9 4 7 1 6 9 1 7 6 6 4 4 9 6 3 5 7 5 4 6 7 3 4 0 3 9 5 0 9 3 6}$ $-26.0000024785177173598094596250259960662517435291393292481504$ $\mathbf{- 3 2 . 0 0 0 0 0 0 0 0 6 8 7 9 9 8 3 8 7 6 5 4 0 7 9 3 9 3 4 2 5 6 4 0 0 5 7 3 8 8 8 6 0 2 0 1 9 7 3 1 8 8 3 4 8 3 7 4 8 9}$ $-48.000000000000000000010590217195128443038701128128133011223$ 0.979413581286539921582714980615301650849208881314033799284678 -18.0664451413826623396696595868981404362417461527291915975943 $\mathbf{0 . 9 4 6 3 5 1 1 8 4 2 4 9 6 6 8 3 1 1 0 6 7 9 5 0 4 7 9 6 1 8 2 1 4 3 5 6 3 3 7 5 8 1 2 4 4 2 5 3 8 5 8 7 1 3 6 2 6 4 1 3}$ $\mathbf{- 3 8 . 0 0 0 0 0 0 0 0 0 0 0 0 0 0 7 7 5 1 6 9 8 1 5 7 0 2 9 4 2 1 3 2 0 5 5 3 7 2 4 3 8 7 4 3 7 7 9 6 4 3 0 9 0 5 5 0 0 3}$ $-\mathbf{6 8 . 0 0 0 0 0 0 0 0 0 0 0 0 0 0 0 0 0 0 0 0 0 0 0 0 0 0 0 0 0 0 0 0 0 0 0 0 0 0 0 0 5 7 8 8 7 7 5 8 8 1 1 8 0 1 6 6 7 8}$ 0.985417671318556372782412173907294420137570423025698038112179 -18.0668262628011359771213953776070300320562537102228077563463 $\mathbf{0 . 9 4 6 3 5 2 2 8 1 4 0 0 7 2 7 0 2 2 3 7 2 3 0 7 7 9 7 0 5 2 4 1 4 9 5 7 3 8 3 5 1 1 0 5 1 3 8 7 5 9 7 3 9 9 8 6 3 5 5 2}$ $-38.0000000000000077517071439509588450710291208956519833987979$ $-68.0000000000000000000000000000000000000000578877588118016678$ $\mathbf{0 . 9 8 5 4 1 7 6 7 1 3 1 8 5 5 6 3 7 2 7 8 2 4 1 2 1 7 3 9 0 7 2 9 4 4 2 0 1 3 7 5 7 0 4 2 3 0 2 5 6 9 8 0 3 8 1 1 2 1 7 9}$ $\mathbf{- 1 8 . 0 6 6 8 2 6 2 6 2 8 0 1 1 3 5 9 7 7 1 2 1 3 9 5 3 7 7 6 0 7 0 3 0 0 3 2 0 5 6 2 5 3 7 1 0 2 2 2 8 0 7 7 5 6 3 4 6 3}$ 0.946352281400727022372307797052414957383511051387597399863552 $\mathbf{- 3 8 . 0 0 0 0 0 0 0 0 0 0 0 0 0 0 7 7 5 1 7 0 7 1 4 3 9 5 0 9 5 8 8 4 5 0 7 1 0 2 9 1 2 0 8 9 5 6 5 1 9 8 3 3 9 8 7 9 7 9}$ $-68.0000000000000000000000000000000000000000578877588118016678$ $\mathbf{0 . 9 8 5 4 1 7 6 7 1 3 1 8 5 5 6 3 7 2 7 8 2 4 1 2 1 7 3 9 0 7 2 9 4 4 2 0 1 3 7 5 7 0 4 2 3 0 2 5 6 9 8 0 3 8 1 1 2 1 7 9}$
\end{abstract}

Figure 10 shows the iterations of the zeta function from the bottom of the list upward leading to the $\operatorname{root} s=-8$ at the top of the list. Note that the iterations seem to originate from a near periodic cycle at the bottom of the list to the root at the top.

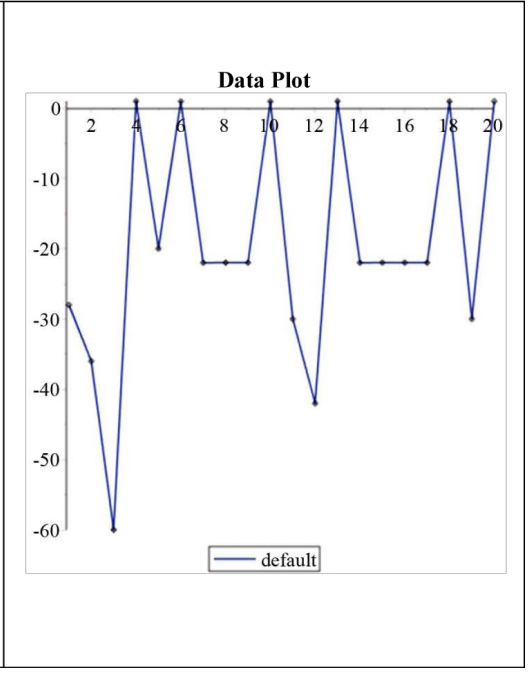

Figure 10. Values of iterations of the root $s=-8$. 
Table 3. Iterations of the arguments below seem to generatealmost periodic cycles of the set below.
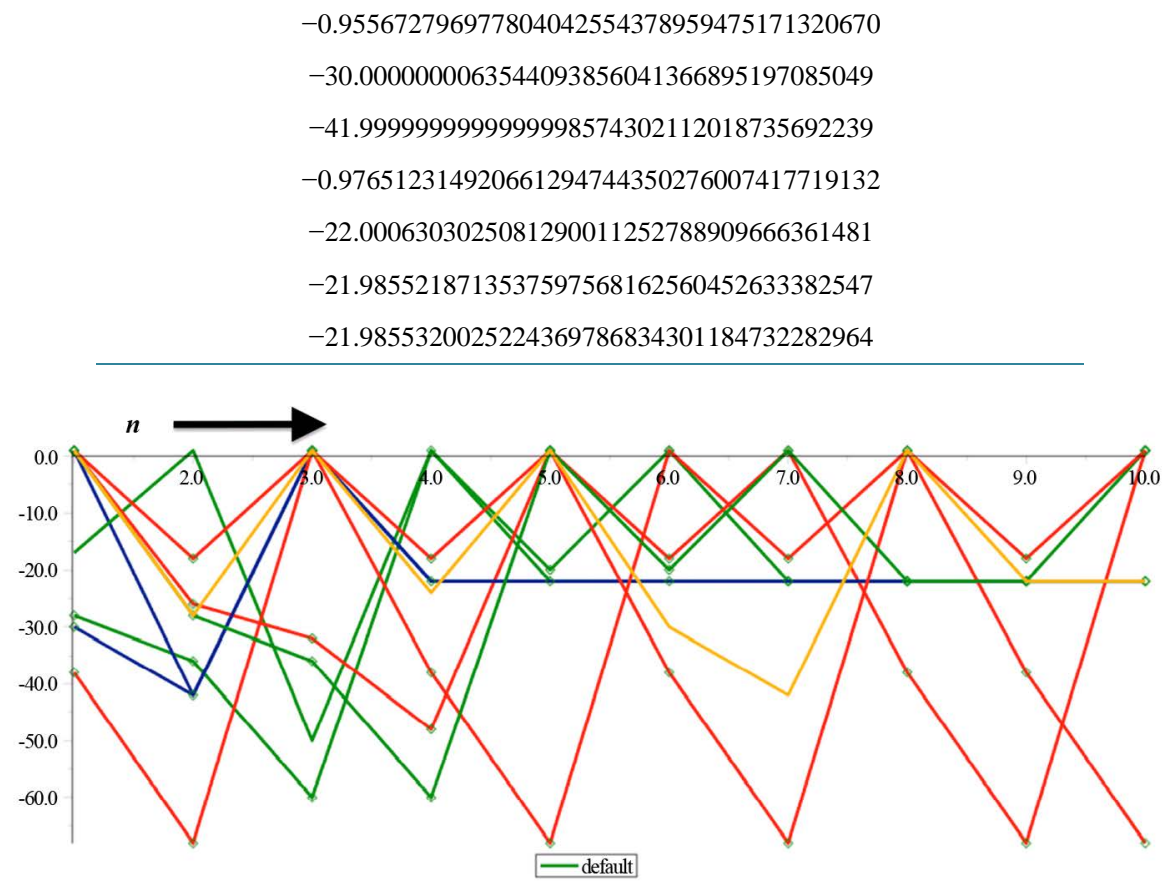

Figure 11. Shows the plots of inverse iterates of real negative roots.

With the general condition that $-\frac{\pi}{2}<\alpha<\frac{\pi}{2}$, these solutions satisfy only the real negative even roots of the function which are the only known real roots of the function. Thus if the roots are on the half-line, the only exponential arguments that will satisfy the roots are for the complex conjugate roots on the half-line. This indicates that the arguments that yield solutions to the vanishing of the Riemann-zeta function are symmetries that satisfy inverse tangent relations and as I will demonstrate in future papers that the arctangent symmetry (223) relates the Bernoulli numbers, Zeta functions, and the Gamma functions to prime numbers.

\section{Discussion of the Result}

The convergence of Power towers relates the vanishing of the zeta function to the half-line. This relationship comes from the property of complex power towers of the exponential-form only converge to exponential functions relating the roots to the convergence. If one iterates backwards from a real root, one finds near misses of purely periodic states of the function as shown in Figures 7-11. Obviously if the cycle ever gets to be purely periodic then no roots can be generated since the periodic cycle will prevent any root from being generated backwards from the infinite past. Table 3 shows the almost periodic cycles of the function that appears to dominate some roots. The inverse iterations seem to generate "very near root" misses.

\section{References}

[1] Borwein, J.M. and Borwein, P.B. (1998) Pi and the AGM. Canadian Mathematical Society Series of Monographs and Advanced Texts, 4. John Wiley \& Sons Inc., New York. A Study in Analytic Number Theory and Computational Complexity; Reprint of the 1987 Original, A Wiley-Interscience Publication.

[2] Corless, R.M., Jeffery, D.J. and Knuth, D. A Sequence of Series for the Lambert W Function. Department of Applied Mathematics, University of Western Ontario London, Canada.

[3] Ehrhardt, W. (2014) The AMath and DAMath Special Functions. Reference Manual and Implementation Notes; Version 2.04, Including Complex Functions. http://creativecommons.org/licenses/by-nc-nd/3.0/

[4] Dence, T.P. (2013) A Brief Look into the Lambert W Function. Applied Mathematics, 4, 887-892. 
http://dx.doi.org/10.4236/am.2013.46122

[5] Shkliarski, D., Chentzov, N. and Yaglom, I. (1993) The USSR Olympiad Problem Book: Selected Problems and Theorems of Elementary Mathematics. Dover Publications, New York.

[6] Anthony, M.M. The Principles of Causal Conspiracy Books 1\&2. Tate Publishing and Enterprises LLC.

[7] Shell, D.L. (1959) Convergence of Infinite Exponentials. University of Cincinnati.

[8] Thron, W.J. (1957) Convergence of Infinite Exponentials with Complex Elements. Proceedings of the American Mathematical Society, 8, 1040-1043. http://dx.doi.org/10.1090/S0002-9939-1957-0096054-X

[9] Galidakis, I.N. (2004) On an Application of Lambert's W Function to Infinite Exponentials. Complex Variables, Theory and Application, 49, 759-780. http://dx.doi.org/10.1080/02781070412331298796

[10] Baker, I.N. and Rippon, P.J. (1985) A Note on Complex Iteration. The American Mathematical Monthly, 92, 501-504. http://www.jstor.org/stable/2322513

[11] Titchmarsh, E.C. FRS; The Theory of the Riemann Zeta Function. Clarendon Press, Oxford University.

[12] Berndt, B.C. (1939) Ramanujan’s Notebooks, Part 1. Springer Varlag.

[13] Sloane, N.J.A. and Plouffe, S. (1995) The Encyclopedia of Integer Sequences. Academic Press.

[14] Vassilev-Missana, M. (2010) Some Results on Infinite Power Towers. Notes on Number Theory and Discrete Mathematics, 16, 18-24. Int. Journal.

[15] Dubickas, A. (2007) On the Powers of Some Transcendental Numbers. Bulletin of The Australian Mathematical Society, 76, 433-440.

[16] Anthony. M.M. A Simple Proof of the Riemann Hypothesis. http://empslocal.ex.ac.uk/people/staff/mrwatkin/zeta/RHproofs.htm

[17] Lester, S.J. a-Points of the Riemann Zeta-Function on the Critical Line. arXiv:1402.0169v1 [math.NT]. 